\title{
Regularization of first order computational homogenization for multiscale analysis of masonry structures
}

\author{
Massimo Petracca - Luca Pelà - Riccardo Rossi • Sergio Oller • Guido \\ Camata • Enrico Spacone
}

Received: date / Accepted: date

\begin{abstract}
This paper investigates the possibility of using classical first order computational homogenization together with a simple regularization procedure based on the fracture energy of the micro-scale-constituents. A generalized geometrical characteristic length takes into account the size of the macro-scale element as well as the size of the RVE (and its constituents).

The proposed regularization ensures objectivity of the dissipated energy at the macro-scale, with respect to the size of the FE in both scales and with respect to the size of the RVE.

The proposed method is first validated against benchmark examples, and finally applied to the numerical simulation of experimental tests on in-plane loaded shear walls made of periodic masonry.
\end{abstract}

Massimo Petracca (西)

CIMNE - Centre Internacional de Metodes Numerics en Enginyeria, Technical University of Catalonia (UPC), Barcelona 08034, Spain

Department of Engineering, University "G.d'Annunzio" of

Chieti and Pescara, Pescara 65127, Italy

E-mail: mpetracca@cimne.upc.edu

E-mail: massimo.petracca@unich.it

Luca Pelà · Riccardo Rossi · Sergio Oller

CIMNE - Centre Internacional de Metodes Numerics en Enginyeria, Technical University of Catalonia (UPC),

Barcelona 08034, Spain

E-mail: luca.pela@upc.edu

E-mail: rrossi@cimne.upc.edu

E-mail: oller@cimne.upc.edu

Guido Camata $\cdot$ Enrico Spacone

Department of Engineering, University "G.d'Annunzio" of Chieti and Pescara, Pescara 65127, Italy

E-mail: g.camata@unich.it

E-mail: espacone@unich.it
Keywords Computational Multiscale Homogenization - Periodic Microstructure - Strain Localization . Characteristic Length · Fracture Energy Regularization · Masonry Shear Wall

\section{Introduction}

The formulation of phenomenological closed-form constitutive laws for the analysis of heterogeneous quasibrittle materials such as masonry is still a challenging task $[45,46]$. The evolution of macroscopic properties is highly influenced by the complex behavior of micro-structural phenomena such as damaged-induced anisotropy, stress redistribution among micro-structural constituents or strain localization. In this context, it is difficult to take into account the influence of the evolving micro-structure on the macroscopic properties, especially when strain localizations occur in the microstructure leading to complex dissipation mechanisms at the structural level.

There are three main approaches to model this kind of heterogeneous materials.

The first approach is the Direct Numerical Simulation (DNS) where a classical 1-scale FE model is used, and all the information about the micro-structure is explicitly modeled. This method provides the best accuracy in taking into account the micro-structure influence on the structural behavior. An interface model using multisurface plasticity has been proposed in [26] to describe the in-plane behavior of masonry. In this kind of modeling, both bricks and mortar joints are explicitly modeled, and all nonlinear behaviors are lumped in the interface elements. This approach is very efficient with small-scale structures (i.e. structural members tested in laboratory). When dealing with large-scale analysis 
the computational cost becomes unaffordable, as well as the effort required to prepare the geometrical model. The second approach is known as Concurrent Multiscale Method (CMM). As a multiscale model, it involves the study of multiple length scales as well as the exchange of information among them. In this particular approach the micro-structural scale is adaptively inserted and resolved on the structural model, thus establishing a strong coupling between macro and micro scales. Works on this subject can be found in [24, 25], among others.

The third approach is known as Computational Homogenization Method (CHM). Similar to the CMM, it is based on multiscale modeling, in that information is obtained from multiple length scales. Assuming that the length scales are separated, this approach establishes a weak coupling between them. The microstructural features are not physically inserted in the structural model, as in the case of CMM, but they are modeled into a so called representative volume element (RVE) which is associated to each integration point of the macroscopic mesh. This RVE, considered as a representative sample of the micro-structure, is then used

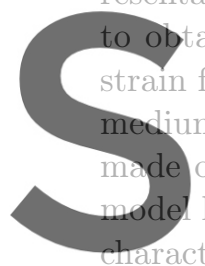
in a homogenized respons ield, thus emulating an m. Due to its flexibility on the homogenized con has been used to model terized by complex micr
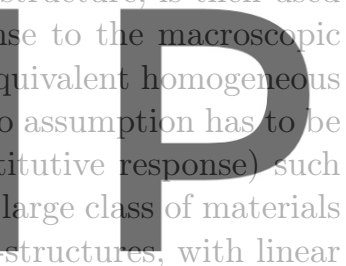
and nonlinear behaviors $[9,41,43,44,47,54,55]$.

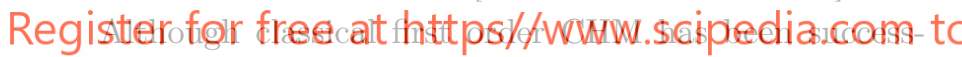
fully used to model micro-structures exhibiting stable behaviors, it has been recognized to give non-objective results when dealing with strain-softening materials. In fact the RVE looses its representativeness upon strain localization. In the last years, several modifications to the classical CHM have been proposed, and a review on recent developments can be found in [35] and in the references therein. Some approaches regularize the response of the RVE by using a higher order theory at the macro-scale, such that the information about a material characteristic length is naturally taken into account $[3,16,17,18,22,23,52]$. Others, known as continuousdiscontinuous approaches, up-scale the RVE response to a traction-separation law (upon strain localization) used by a discontinuity inserted into the macro-scale model [6, 8, 30, 31, 32, 33, 37, 39]. Another issue related to the loss of representativeness of the RVE is the choice of proper boundary conditions to be applied on the RVE. A study on this topic has been done in [13], where a novel boundary condition has been proposed, using the concept of rotating periodicity to avoid costraining the crack propagation orientation.
In the context of masonry modeling, works on continuousdiscontinuous computational homogenization can be found in $[30,35]$ for modeling the in-plane behavior of masonry structures, and later on extended to the study of shell elements for the analysis of the out-of-plane behavior [32, 33], while works on computational homogenization using a Cosserat continuum at the macro-scale can be found in $[16,17]$.

Previous approaches based on first order homogenization and fracture energy regularization considered the characteristic length used in the microstructural constitutive models equal to the characteristic length of the macro-scale finite elements [4]. The same was done in [16] to compare the classical first order homogenization with the Cosserat-based homogenization. This paper proposes an extension of the fracture energy-based regularization to two-scale computational homogenization based on classical first order continuum theory. The main aim is to allow the usage of first order computational homogenization for the analysis of quasi-brittle micro-structures. The main novelty of the proposed approach resides in the consistent definition of a frac-

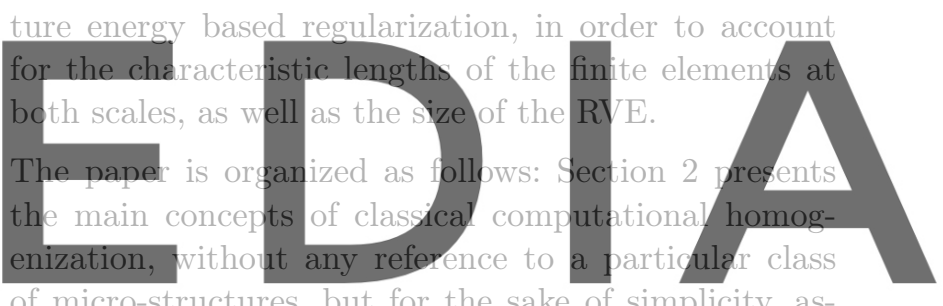

of micro-structures, but for the sake of simplicity, assuming small strain theory. In Section 3, the issues indowdiond the versign withqut thewatermark

and the proposed regularization procedure is derived. In Section 4, a first benchmark test is used to assess the proposed regularization with respect to a simple mode I fracture test. Finally in Section 5, attention is given to the modeling of masonry structures, through a more complicated test on in-plane loaded shear walls.

\section{Classical first order computational homogenization}

This section gives a brief summary of the main concepts and basic equations of classical first order computational homogenization, where a standard first order continuum theory is assumed in both scales. In the present work we assume the infinitesimal strain theory.

The main work-flow of the classical CHM can be represented as in Figure 1. 


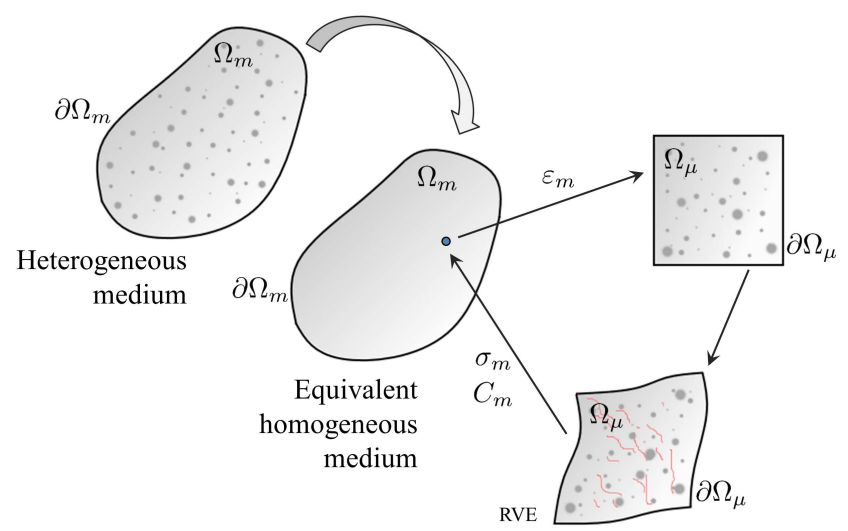

Fig. 1: work-flow of classical computational homogenization

The procedure can be defined by three main steps:

1. Down-scaling or macro-micro transition, where the macroscopic strain at any point of the macroscopic mesh, are transferred to the micro-scale, where they are used to apply boundary conditions on the RVE mesh;

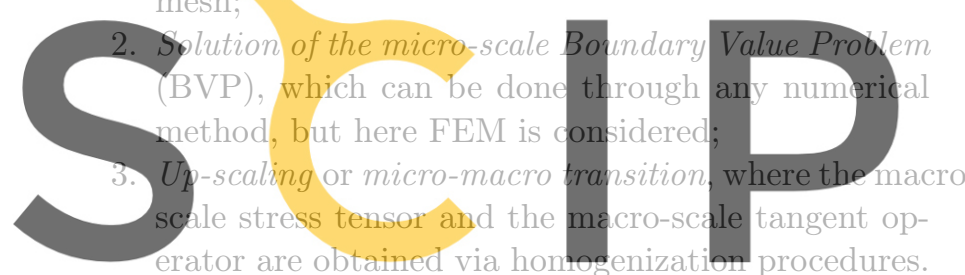

and in the same way the microscopic strain field can be split into a constant part (coarse scale contribution) and a fluctuating part $\tilde{\varepsilon}_{\mu}$ (fine scale contribution)

$\boldsymbol{\varepsilon}_{\mu}\left(\boldsymbol{x}_{\mu}, t\right)=\boldsymbol{\varepsilon}_{m}\left(\boldsymbol{x}_{m}, t\right)+\tilde{\varepsilon}_{\mu}\left(\boldsymbol{x}_{\mu}, t\right)$

Inserting the definition of the microscopic strain field (Eq. (3)) into the definition of the macroscopic strain field (Eq. (1)), one obtains:

$\boldsymbol{\varepsilon}_{m}\left(\boldsymbol{x}_{m}, t\right)=\varepsilon_{m}\left(\boldsymbol{x}_{m}, t\right)+\frac{1}{V_{\mu}} \int_{\Omega_{\mu}} \nabla^{s} \tilde{\boldsymbol{u}}_{\mu} \mathrm{d} V$

which provides the minimal kinematic constraint that a microscopic displacement fluctuation field should satisfy to be kinematically admissible:

$\int_{\Omega_{\mu}} \nabla^{s} \tilde{\boldsymbol{u}}_{\mu} \mathrm{d} V=\int_{\partial \Omega_{\mu}} \tilde{\boldsymbol{u}}_{\mu} \otimes_{s} \boldsymbol{n} \mathrm{d} A=0$

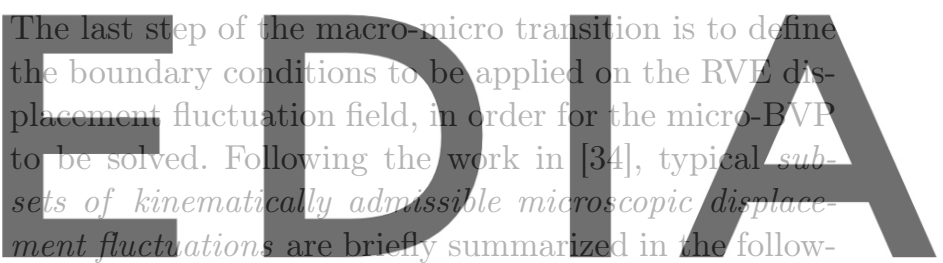

ing.

Register for free at https/_www scipedia.com to downleaglathemersign without the watermark

The macroscopic strain $\varepsilon_{m}$, in each point $x_{m}$ of the macro-scale domain and at each instant $t$, can be obtained as the volume average of the microscopic strain field $\varepsilon_{\mu}$ defined at each point $\boldsymbol{x}_{\mu}$ of the micro-scale domain and at each instant $t$ :

$$
\begin{aligned}
\boldsymbol{\varepsilon}_{m}\left(\boldsymbol{x}_{m}, t\right) & =\frac{1}{V_{\mu}} \int_{\Omega_{\mu}} \boldsymbol{\varepsilon}_{\mu}\left(\boldsymbol{x}_{\mu}, t\right) \mathrm{d} V \\
& =\frac{1}{V_{\mu}} \int_{\Omega_{\mu}} \nabla^{s} \boldsymbol{u}_{\mu} \mathrm{d} V \\
& =\frac{1}{V_{\mu}} \int_{\partial \Omega_{\mu}} \boldsymbol{u}_{\mu} \otimes_{s} \boldsymbol{n} \mathrm{d} A
\end{aligned}
$$

being $\boldsymbol{n}$ the outward unit normal field on the RVE boundary $\partial \Omega_{\mu}$. It is assumed that the microscopic displacement field can be additively split into a linear part (coarse scale contribution) and a fluctuating part $\tilde{\boldsymbol{u}}_{\mu}$ (fine scale contribution)

$\boldsymbol{u}_{\mu}\left(\boldsymbol{x}_{\mu}, t\right)=\varepsilon_{m}\left(\boldsymbol{x}_{m}, t\right) \boldsymbol{x}_{\mu}+\tilde{\boldsymbol{u}}_{\mu}\left(\boldsymbol{x}_{\mu}, t\right)$ $\tilde{u}_{\mu}\left(x_{\mu}, t\right)=0 \quad \forall x_{\mu} \in \Omega_{\mu}$

Displacement fluctuations vanish in every point of the RVE domain, thus the whole RVE undergoes a uniform strain equal to the macro-scale strain field.

- Zero boundary displacement fluctuations

$\tilde{\boldsymbol{u}}_{\mu}\left(\boldsymbol{x}_{\mu}, t\right)=0 \quad \forall \boldsymbol{x}_{\mu} \in \partial \Omega_{\mu}$

Displacement fluctuations vanish only on the boundaries of the RVE.

- Periodic boundary displacement fluctuations

$\tilde{\boldsymbol{u}}_{\mu}\left(\boldsymbol{x}_{\mu}^{+}, t\right)=\tilde{\boldsymbol{u}}_{\mu}\left(\boldsymbol{x}_{\mu}^{-}, t\right) \quad \forall \operatorname{pair}\left\{\boldsymbol{x}_{\mu}^{+}, \boldsymbol{x}_{\mu}^{-}\right\} \in \partial \Omega_{\mu}$

being $\boldsymbol{x}_{\mu}^{+}$and $\boldsymbol{x}_{\mu}^{-}$two opposite points on the RVE boundary $\partial \Omega_{\mu}$. This model constraints the displacement fluctuations on the boundaries of the RVE to be periodic. 
- Minimally constrained boundary displacement fluctuations or Uniform boundary traction

$\boldsymbol{\sigma}_{\mu}\left(\boldsymbol{x}_{\mu}, t\right) \boldsymbol{n}\left(x_{\mu}\right)=\boldsymbol{\sigma}_{m}\left(\boldsymbol{x}_{m}, t\right) \boldsymbol{n}\left(x_{\mu}\right) \quad \forall \boldsymbol{x}_{\mu} \in \partial \Omega_{\mu}$

Where $\boldsymbol{\sigma}_{\mu}\left(\boldsymbol{x}_{\mu}, t\right)$ is the microscopic stress field. As shown in [34], the distribution of stresses on the RVE boundaries, reactive to the minimal kinematic constraint given in Eq. (5), satisfies Eq. (9)

\subsection{Solution of the micro-scale BVP}

Assuming that inertia forces are negligible, the Principle of Virtual Work establishes that the RVE is in equilibrium if and only if the variational equation

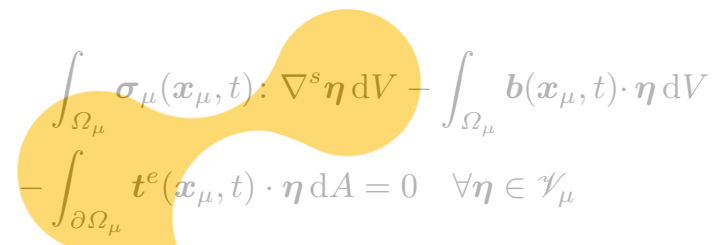

holds at each instant $t$. In Eq. (10), $b\left(x_{\mu}, t\right)$ is the body

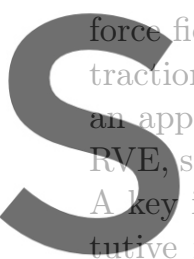

din the RVE domain,

raction field on the boundaries

opriate space of virtua

ingredient in the definition

models is the Hill-Mande
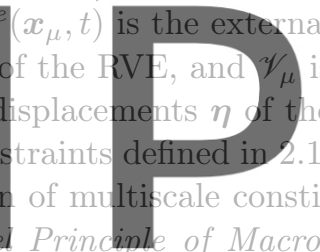

Homogeneity [20, 29], an energy average theorem es-

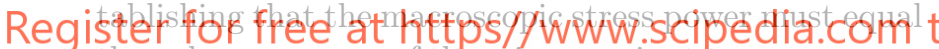

the volume average of the microscopic stress power over the RVE

$\boldsymbol{\sigma}_{m}: \dot{\varepsilon}_{m}=\frac{1}{V_{\mu}} \int_{\Omega_{\mu}} \boldsymbol{\sigma}_{\mu}: \dot{\varepsilon}_{\mu} \mathrm{d} V$

for any kinematically admissible microscopic strain rate field $\dot{\varepsilon}_{\mu}$, i.e. (following the additive split between coarse scale and fine scale contributions) for any microscopic strain rate field of the form:

$\dot{\boldsymbol{\varepsilon}}_{\mu}=\dot{\boldsymbol{\varepsilon}}_{m}+\nabla^{s} \dot{\tilde{\boldsymbol{u}}}_{\mu} \quad \forall \dot{\tilde{\boldsymbol{u}}}_{\mu} \in \mathscr{V}_{\mu}$

As proved in [34], the Hill-Mandel Principle is equivalent to requiring that body forces and surface traction in the RVE be purely reactive, i.e. they are reaction to the chosen kinematic constraints, and cannot be prescribed independently. In this way the RVE equilibrium reduces to:

$\int_{\Omega_{\mu}} \boldsymbol{\sigma}_{\mu}\left(\boldsymbol{x}_{\mu}, t\right): \nabla^{s} \boldsymbol{\eta} \mathrm{d} V=0$

\subsection{Up-scaling or micro-macro transition}

The last step in the homogenization procedure consists in finding the homogenized macroscopic stress tensor (and if required the homogenized tangent operator) after the RVE equilibrium is found. As shown in [19, 42], a definition of the homogenized stress tensor can be derived directly from the Hill-Mandel Principle and the additive split of the microscopic strain, and not as an assumption of the model. Inserting the split of the microscopic strain rate given in Eq. (12), into the Hill-Mandel Principle Eq. (11), the following relation is obtained:

$$
\begin{aligned}
\boldsymbol{\sigma}_{m}: \dot{\boldsymbol{\varepsilon}}_{m} & =\frac{1}{V_{\mu}} \int_{\Omega_{\mu}} \boldsymbol{\sigma}_{\mu}: \dot{\varepsilon}_{m} \mathrm{~d} V \\
& +\frac{1}{V_{\mu}} \int_{\Omega_{\mu}} \sigma_{\mu}: \nabla^{s} \dot{\tilde{u}}_{\mu} \mathrm{d} V \quad \forall \dot{\tilde{u}}_{\mu} \in \mathscr{V}_{\mu}
\end{aligned}
$$

which is valid for any kinematically admissible $\dot{\tilde{u}}_{\mu}$, and for the case of $\dot{\tilde{u}}_{\mu}=0$, the following relation is obtained:

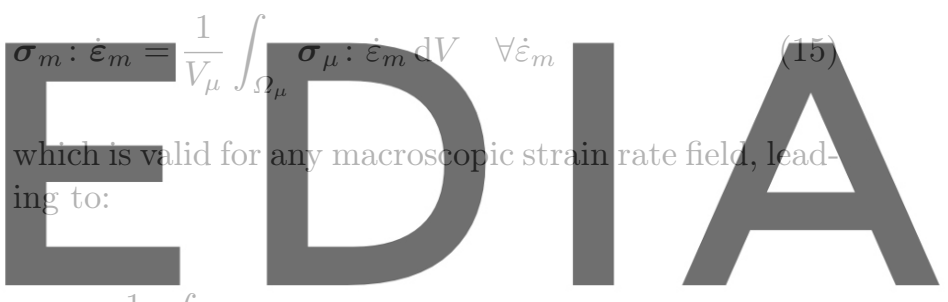

$\sigma_{m}=\frac{1}{V_{\mu}} \int \sigma_{\mu}\left(x_{\mu}, t\right) \mathrm{d} V$

(16)

\section{download the version without the watermark}

Thus the homogenized macroscopic stress tensor can be obtained as the volume average of the microscopic stress field of the RVE.

2.4 Interpretation and comparison with other multiscale methods

As already mentioned in Section 1, CHM allows a straightforward interpretation within the framework of Variational Multiscale Methods (VMM). The main idea is to consider that the continuous displacement field $\boldsymbol{u}$ can be decomposed into a coarse and a fine component as

$\boldsymbol{u}=\overline{\boldsymbol{u}}+\tilde{\boldsymbol{u}}$

where $\overline{\boldsymbol{u}}$ is defined as the "coarse" scale contribution (i.e. the one described by the coarse FE mesh) and $\tilde{\boldsymbol{u}}$ is the "fine" scale contribution (i.e. the one that would correspond to a detail that cannot be solved by the coarse FE mesh). The corresponding test functions are 
named $\bar{w}$ and $\tilde{w}$. Using such decomposition the strain $\varepsilon:=\nabla^{s} \boldsymbol{u}$ becomes

$\varepsilon=\nabla^{s} \overline{\boldsymbol{u}}+\nabla^{s} \tilde{\boldsymbol{u}}$

To follow the standard multiscale procedure [21], one shall at this point introduce a model to obtain $\tilde{\boldsymbol{u}}$. This can be done by applying the Galerkin approach at the level of the fine scale to give

$\int_{\Omega} \tilde{w} \nabla \cdot \boldsymbol{\sigma}\left(\nabla^{s} \tilde{\boldsymbol{u}}\right) \mathrm{d} V=\int_{\Omega} \tilde{w} \boldsymbol{f} \mathrm{d} V-\int_{\Omega} \tilde{w} \nabla \cdot \boldsymbol{\sigma}\left(\nabla^{s} \overline{\boldsymbol{u}}\right) \mathrm{d} V$

which defines a "fine" scale problem which can be eventually solved if one, as in our case, provides a suitable discretization of the fine scale domain (that is, a way to actually compute $\tilde{w}$ ). The problem is completed once one prescribes a suitable Boundary Condition for $\tilde{u}$. In the VMM community it is customary to assume that

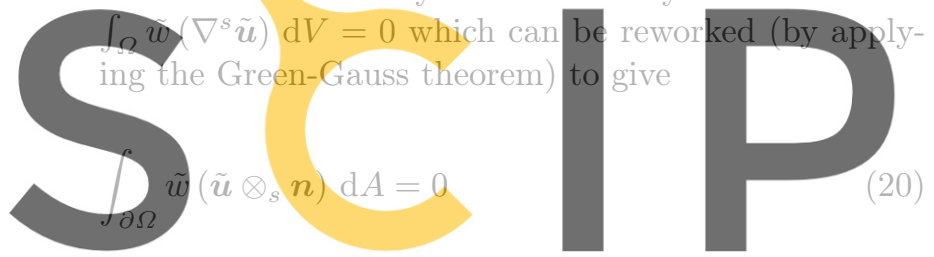

In the case of CHM, $\tilde{w}$ can be considered as a discontin-

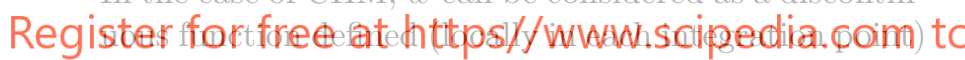
by the Finite Element discretization of the RVE. Under this assumption, Eq. (19) and Eq. (20) can be restricted to the RVE volume to give the Finite Element problem

$\int_{\Omega_{R V E}} \tilde{w} \nabla \cdot \boldsymbol{\sigma}\left(\nabla^{s} \tilde{\boldsymbol{u}}\right) \mathrm{d} V=$

$\int_{\Omega_{R V E}} \tilde{w} \boldsymbol{f} \mathrm{d} V-\int_{\Omega_{R V E}} \tilde{w} \nabla \cdot \boldsymbol{\sigma}\left(\nabla^{s} \overline{\boldsymbol{u}}\right) \mathrm{d} V$

$\int_{\partial \Omega_{R V E}} \tilde{w}\left(\tilde{\boldsymbol{u}} \otimes_{S} \boldsymbol{n}\right) \mathrm{d} A=0$

Interestingly, Eq. (22) coincides with the so called "minimal" boundary conditions for $\tilde{\boldsymbol{u}}$. Observe that both $\tilde{\boldsymbol{u}}=0$ (in $\partial \Omega_{R V E}$ ) and the constraint of $\tilde{\boldsymbol{u}}$ being periodic (in $\partial \Omega_{R V E}$ ) are special cases that comply with this boundary condition. Observe also that no assumption on the periodicity of $\tilde{\boldsymbol{u}}$ is needed when deriving minimal boundary conditions.

\section{Fracture-energy-based regularization in two-scale FE Computational Homogenization}

An essential aspect in numerical modeling is mesh objectivity, defined as the requirement that the results must be independent of the adopted discretization.

In a continuum which exhibits strain-softening, the strain localizes into a narrow zone where energy is dissipated according to its size, while outside the material unloads elastically. On the other hand, in the FEM context, if standard local continuum models are used (i.e. the material response is computed as a function of local strains), this localization zone is related to the domain (volume or area) of each finite element. Thus, if strain softening appears, the dissipative domain will coincide with only one strip of elements through the finite element mesh, but since the dissipation of the total energy is proportional to the size of the finite elements, the energy dissipated would diminish as the elements become smaller upon mesh refinement, leading to non-objective results that depend on the FE size.

A solution to this problem, now widely used, has been proposed first in [5], and later on addressed by several

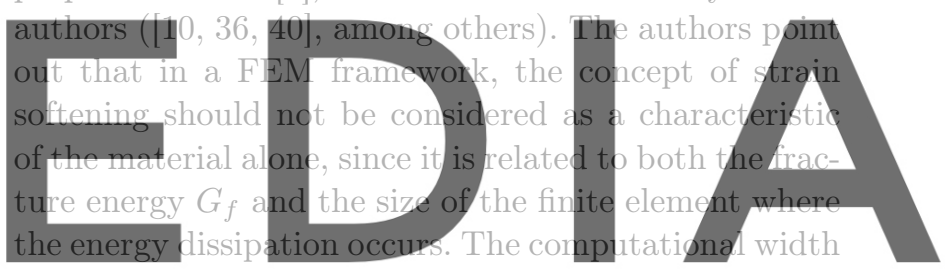

of the fracture process is computed in each element de-

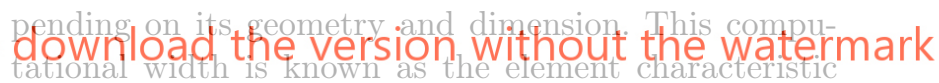
length $l_{c h}$. According to this model, the softening law depends on both the material fracture energy per unit of crack surface area $G_{f}$ and the element characteristic length $l_{c h}$, in such a way that the following relation holds:

$G_{f}=\frac{W}{A}=\frac{W}{V} l_{c h}=g_{f} l_{c h}$

where $W$ is the total energy dissipated by the fracture process, $A$ is the crack surface area, $g_{f}$ is the fracture energy per unit of volume, and $V$ is the volume of the dissipative zone.

Referring to the notations given in Figure 2, Eq. (23) can be rewritten as:

$G_{f}=\frac{W}{h t}=\frac{W}{h t l_{c h}} l_{c h}=g_{f} l_{c h}$

In two-scale FE analysis, the concept of characteristic length of the parent element (the element containing the constitutive law that needs to be regularized) 


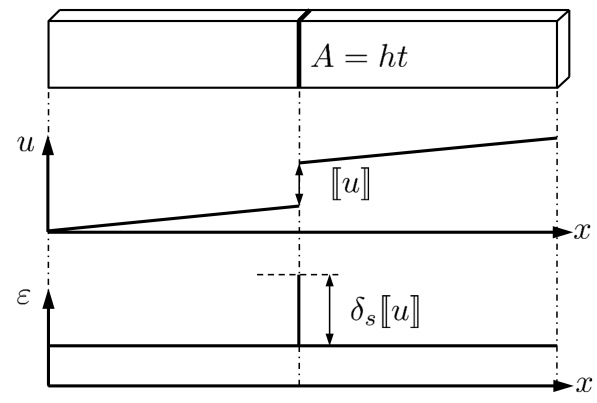

(a) strong discontinuity
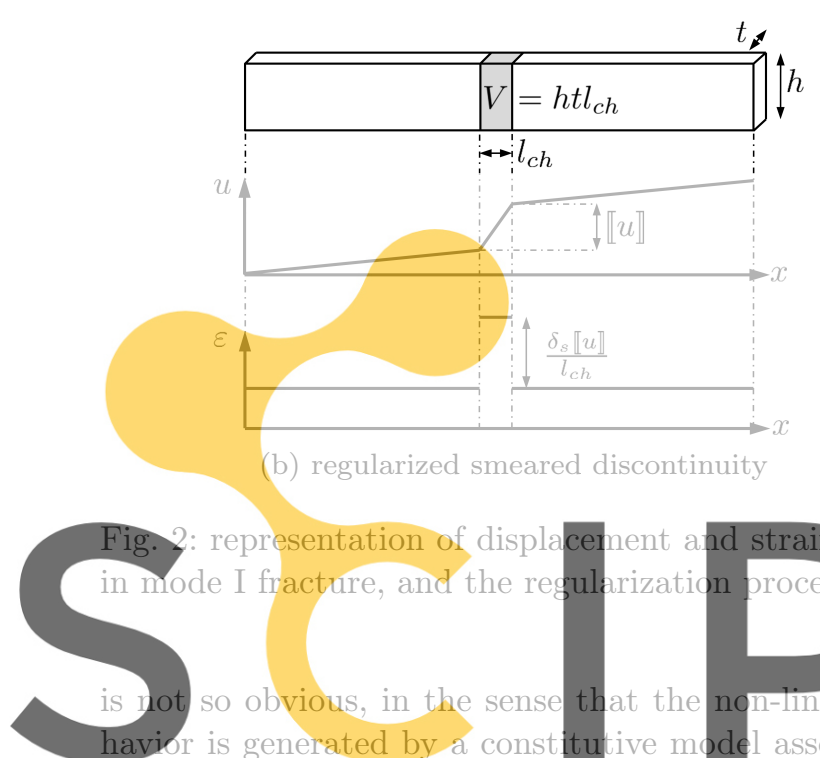

representation of displaces
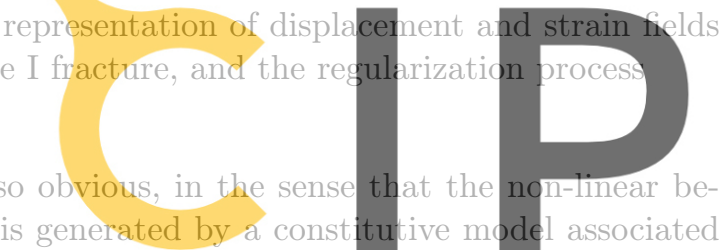

to a domain of the micro-scale, but the dissipation fi-

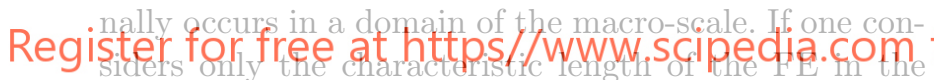

micro-scale, one would obtain a correct dissipation in the RVE, but then, changing the size of the RVE or the size of the FE at the macro-scale, one would obtain non-objective responses at the macro-scale. Both requirements of mesh-objectivity and correct dissipation can be satisfied only if a regularization is employed in both scales of the analysis.

This suggests that the regularization parameter for the constitutive model used in the micro-scale should be adjusted to take into account the nested feature of the multiscale homogenization procedure. As a starting point we can assume that the constitutive law at the microscale should be regularized using a modified characteristic length $\widetilde{l}_{c h, \mu}$, defined as a function of all the characteristic lengths that may enter into the problem (see Figure 3):

$\tilde{l}_{c h, \mu}=f\left(l_{c h, \mu}, l_{c h, m}, l_{c h, R V E}\right)$

where $l_{c h, \mu}$ is the characteristic length of the FE in the micro-scale, $l_{c h, m}$ is the characteristic length of the FE in the macro-scale, and $l_{c h, R V E}$ is the characteristic length of the RVE itself. The characteristic length of the FE (both at the micro and at the macro scale) has to be always computed according to the geometry, interpolation and integration scheme of the considered finite element [49].
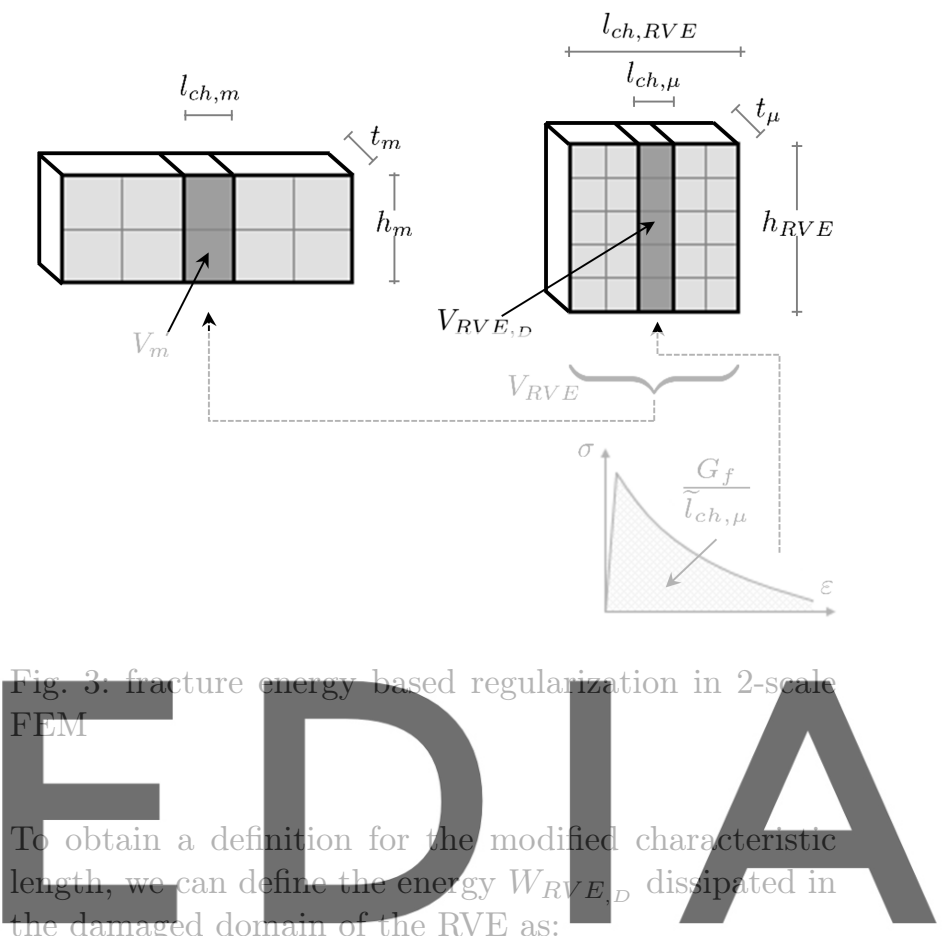

\section{to do}

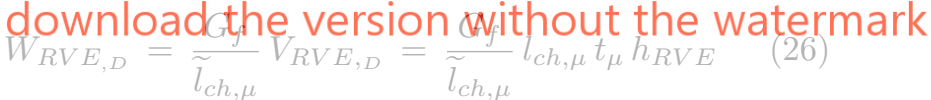

where $V_{R V E,{ }_{D}}$ is the damaged domain of the RVE, $h_{R V E}$ is the length of the RVE in the direction of the crack, and $t_{\mu}$ is the thickness of the RVE. We can now "smear" the dissipated energy over the whole RVE domain, defining an equivalent specific fracture energy $\widetilde{g}_{f}$ as:

$$
\begin{aligned}
\widetilde{g}_{f}= & \frac{W_{R V E, D}}{V_{R V E}}=\frac{W_{R V E, D}}{l_{c h, R V E} h_{R V E} t_{\mu}}= \\
& \frac{G_{f}}{\widetilde{l}_{c h, \mu}} \frac{l_{c h, \mu}}{l_{c h, R V E}}
\end{aligned}
$$

Integrating this specific fracture energy over the dissipating domain at the macro-scale we get the total energy $W_{m}$ dissipated at the macro-scale:

$W_{m}=\int_{A_{m}} \widetilde{g}_{f} \mathrm{~d} V_{m}$

$W_{m}=\widetilde{g}_{f} l_{c h, m} h_{m} t_{m}$ 
Where $h_{m}$ and $t_{m}$ are respectively the length and the thickness of the crack at the macro-scale. Substituting Eq. (27) in Eq. (29), and equating it with the actual dissipated energy that we're seeking at the structural level

$$
W=G_{f} h_{m} t_{m}
$$

we obtain a definition for the modified characteristic length, to be used at the micro-scale, that provides the proper dissipation at the macro-scale:

$$
W_{m}=\frac{G_{f}}{\widetilde{l}_{c h, \mu}} \frac{l_{c h, \mu}}{l_{c h, R V E}} l_{c h, m} h_{m} t_{m}=G_{f} h_{m} t_{m}
$$

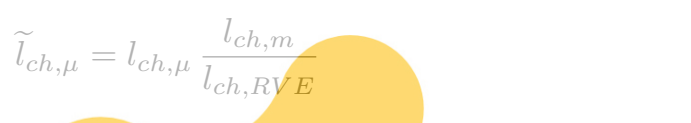

From Eq. (32) it can be seen that the characteristic lengths at the micro-scale should be multiplied by a scaling factor (constant over the RVE) which is the ratio between the characteristic length of the parent-element at the macro-scale and the characteristic length of the
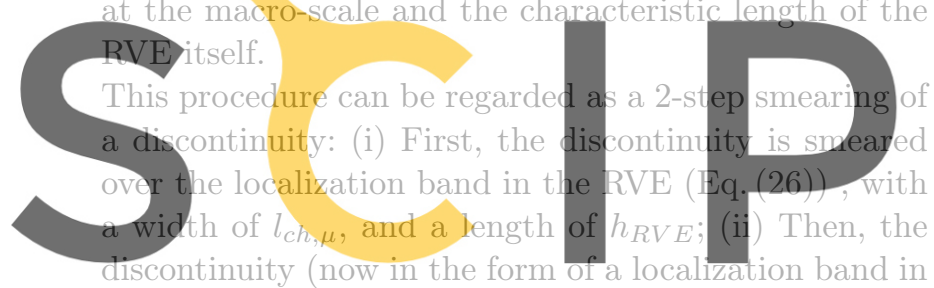

the RVE) is smeared over the localizing element at the

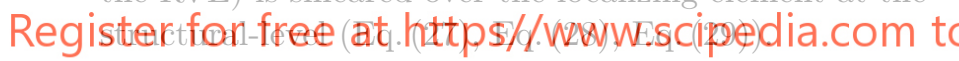

In [4], the modified characteristic length to be used at the micro-scale was taken equal to the characteristic length of the macro-scale FE. In that context, however, the author was dealing with composites made of damageable matrix and long fibers. Long fibers are typically oriented along the loading direction, allowing damage to spread over a wide area. In those cases damage is distributed over the whole RVE domain, while the localization may happen at the structural level. On the contrary, in masonry the localization is already visible in the RVE, thus requiring to consider both length scales during the regularization procedure. It should be noted however, that the method proposed in [4] is recovered from Eq. (32), setting $l_{c h, \mu}=l_{c h, R V E}$.

In [31] the localized RVE was homogenized towards a discontinuity with a band width equal to the RVE characteristic length. In the approach proposed by this work, this corresponds to $l_{c h, m}=l_{c h, R V E}$, thus leaving the characteristic length of the microstructural elements unchanged.

Eq. (26) defines the total energy dissipated in the damaged part of the RVE, assuming that the total length of the crack is equal to the length of the RVE in the crack direction $\left(h_{R V E}\right)$, thus assuming a straight crack. Let's assume instead that in the RVE the crack is nonstraight, with a total length equal to $\Phi h_{R V E}$, where $\Phi=1$ is a scaling factor that measures the tortuosity of the crack. Eq. (26) then becomes

$$
W_{R V E, D}=\frac{G_{f}}{\widetilde{l}_{c h, \mu}} l_{c h, \mu} t_{\mu} \Phi h_{R V E}
$$

Eq. (30), refers to the total energy dissipated by the real crack that we should simulate, and it was assumed straight (as for the crack in the RVE) with a length equal to the length of the specimen in the crack direction $h_{m}$. If we consider instead a non-straight crack, Eq. (30) becomes

$W=G_{f} \Phi h_{m} t_{m}$

\section{Thus Eq. (31) becomes}

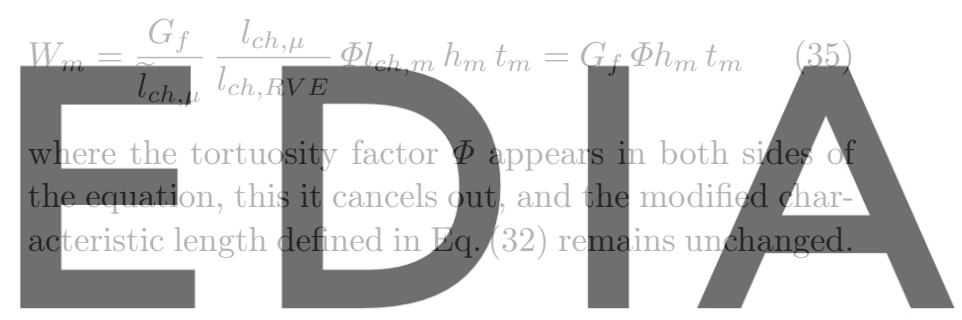

\section{Mode I fracture test}

\section{download the version without the watermark}

The test reported in this section is meant to assess the regularization capabilities of the proposed method with respect to a simple mode I fracture test. The specimen is a rectangular plate in a state of plane-stress subject to a uniaxial tensile load. The micro-structure is assumed as a matrix with a periodic pattern of circular voids (note that voids are emulated with a very soft linear elastic material as reported in Table 1). The regularization with respect to the macro-scale FE size and the size of the RVE is checked by performing the same analysis on two different macro-scale discretizations and two different RVE sample sizes, for a total of four analyses. The same analyses are conducted with and without the regularization procedure to better show the differences. Details about the geometry, the two macro-scale models, and the two RVEs, are given respectively in [Figure 4, Figure 5, Figure6], while details about the mechanical properties of the micro-structural constituents are given in Table 1. Periodic boundary displacement fluctuations are employed in the multiscale analysis, since in this example we expect cracks aligned with the periodicity directions. 
A local continuum damage model is used to describe the constitutive behavior of the matrix in the RVE. The main concepts of the model are summarized here. For further details the reader can refer to $[10,12]$ and the references therein. The constitutive equation is defined as

$\boldsymbol{\sigma}=(1-d) \overline{\boldsymbol{\sigma}}=(1-d) \boldsymbol{C}: \boldsymbol{\varepsilon}$

where the stress tensor $\boldsymbol{\sigma}$ is obtained as a function of the effective stress tensor $\overline{\boldsymbol{\sigma}}$, computed from the total strain tensor $\varepsilon$ and the fourth order isotropic linear elastic constitutive tensor $\boldsymbol{C}$, and the damage index $d$, which ranges from zero for the undamaged material, to one for the completely damaged material. In order to compute this damage index, related to the current effective stress state, it is necessary to introduce a scalar measure, termed as equivalent stress $\tau$. In this example we assume a Rankine criterion, so the equivalent stress can be computed as follows:

$\tau=\left[\bar{\sigma}^{+}: \Lambda: \bar{\sigma}^{+}\right]^{1 / 2}$
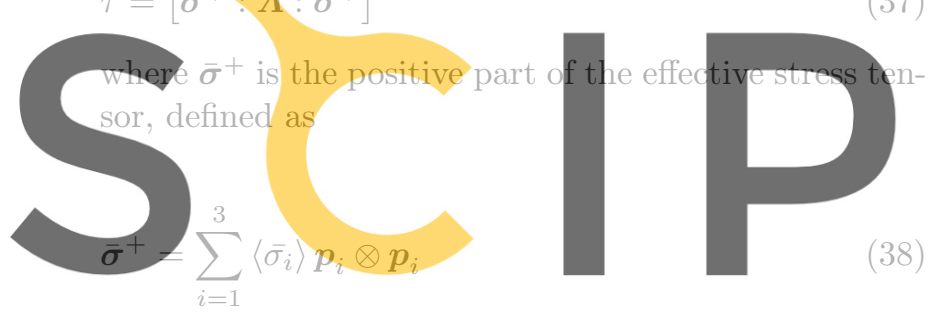

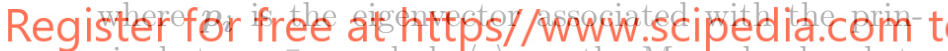
cipal stress $\bar{\sigma}_{i}$, symbols $\langle\bullet$ are the Macaulay brackets $(\langle x\rangle=x$, if $x \geq 0,\langle x\rangle=0$, if $x<0)$, and $\Lambda$ is a non-dimensional fourth order tensor defined as

$$
\boldsymbol{\Lambda}=\boldsymbol{p}_{1} \otimes \boldsymbol{p}_{1} \otimes \boldsymbol{p}_{1} \otimes \boldsymbol{p}_{1}
$$

Being the damage an irreversible process, we can introduce another scalar quantity, termed as damage threshold $r$, that represents the largest value ever reached by the equivalent stress $\tau$ during the loading history. The damage threshold $r$ at time $t+\Delta t$ can be evaluated as

$r=\max \left(r_{0}, \max \left(r_{t}, \tau\right)\right)$

where $r_{t}$ is the damage threshold at the previous (converged) time step, and $r_{0}$ is the initial damage threshold (the one that defines the elastic limit) that in this case is $r_{0}=f_{t}$. Accordingly, the following damage criterion is introduced:

$\Phi(\tau, r)=\tau-r_{t} \leq 0$
In this way, the damage index $d$, in the time interval $[t, t+\Delta t]$, can grow only if $\tau>r_{t}$. In this test the damage is assumed to evolve according to the following exponential law:

$$
d(r)=1-\frac{r_{0}}{r} \exp \left\{2 H_{d i s}\left(\frac{r_{0}-r}{r_{0}}\right)\right\}
$$

where $H_{d i s}$ is the discrete softening parameter. As described in Section 3, in the discrete problem the softening law should be adjusted according to the size of the dissipative zone $\left(l_{d i s}\right)$, in such a way that the following equation holds:

$g_{f} l_{d i s}=G_{f}$

where the specific dissipated energy $g_{f}$, for the exponential softening law, can be calculated as:

$$
g_{f}=\left(1+\frac{1}{H_{d i s}}\right) \frac{f_{t}^{2}}{2 E}
$$

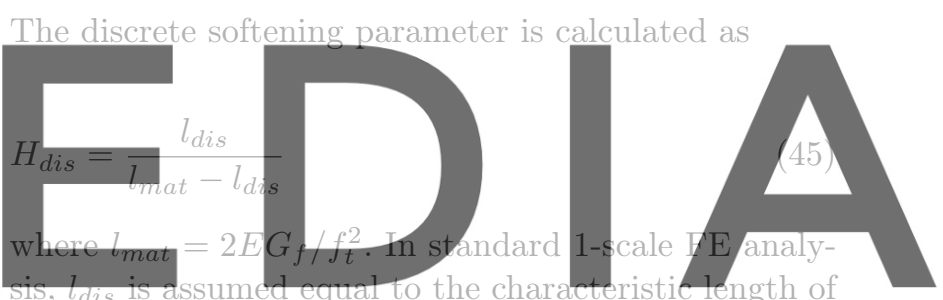

the FE $\left(l_{d i s}=l_{c h}\right)$. Here we are using this constitutive

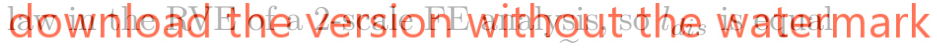
to the modified characteristic length $l_{c h, \mu}$, as defined in Eq. (32).

The analysis has been performed using Kratos Multiphysics [14], a free open-source framework for the development of multidisciplinary solvers, developed at CIMNE, while pre and post-processing have been done in GiD [1], also developed at CIMNE.

The results obtained without the proposed regularization procedure (Figure 7a) show four widely distinct force-displacement curves. As expected, the response is more brittle for larger RVEs and/or with finer meshes at the macro-scale. Applying the proposed regularization all the analyses give the same response (Figure $7 \mathrm{~b}$ ), thus showing objectivity with respect to the macro-scale FE size and to the RVE size. The macro-scale displacement field and the micro-scale maximum principal strain, at the end of the analysis, are shown in Figure 8. 
Regularization of first order computational homogenization for multiscale analysis of masonry structures

\begin{tabular}{cccc}
\hline Property & Value (Material 1) & Value (Material 2) & Unit \\
\hline \hline Young's modulus & 14000.0 & 1.0 & $\mathrm{~N} / \mathrm{mm}^{2}$ \\
\hline Poisson's ratio & 0.15 & 0.15 & - \\
\hline Type & Continuum damage model & Linear elastic & - \\
\hline Tensile maximum stress & 2.2 & - & $\mathrm{N} / \mathrm{mm}^{2}$ \\
\hline Tensile fracture energy & 0.8 & - & $\mathrm{N} / \mathrm{mm}$ \\
\hline Damage criterion & Rankine & - & - \\
\hline Softening law & Exponential & - & - \\
\hline
\end{tabular}

Table 1: material properties
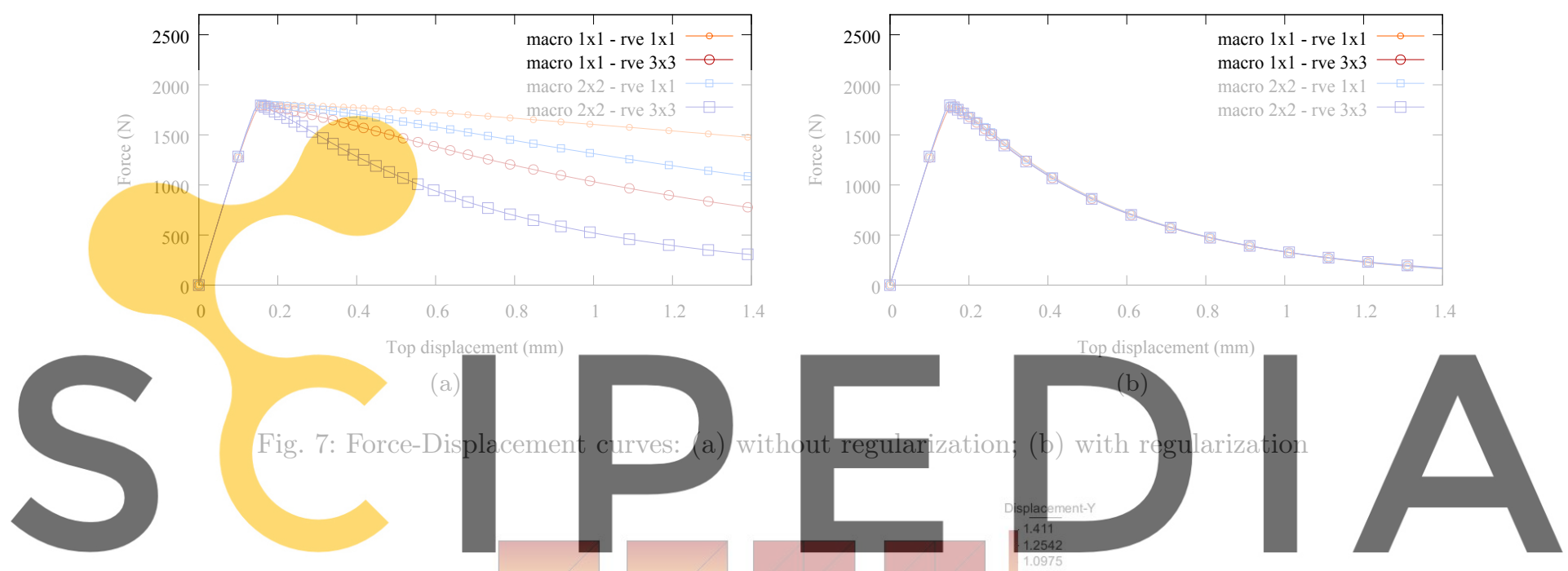

Register for free at https//www.scipedia:com to download the
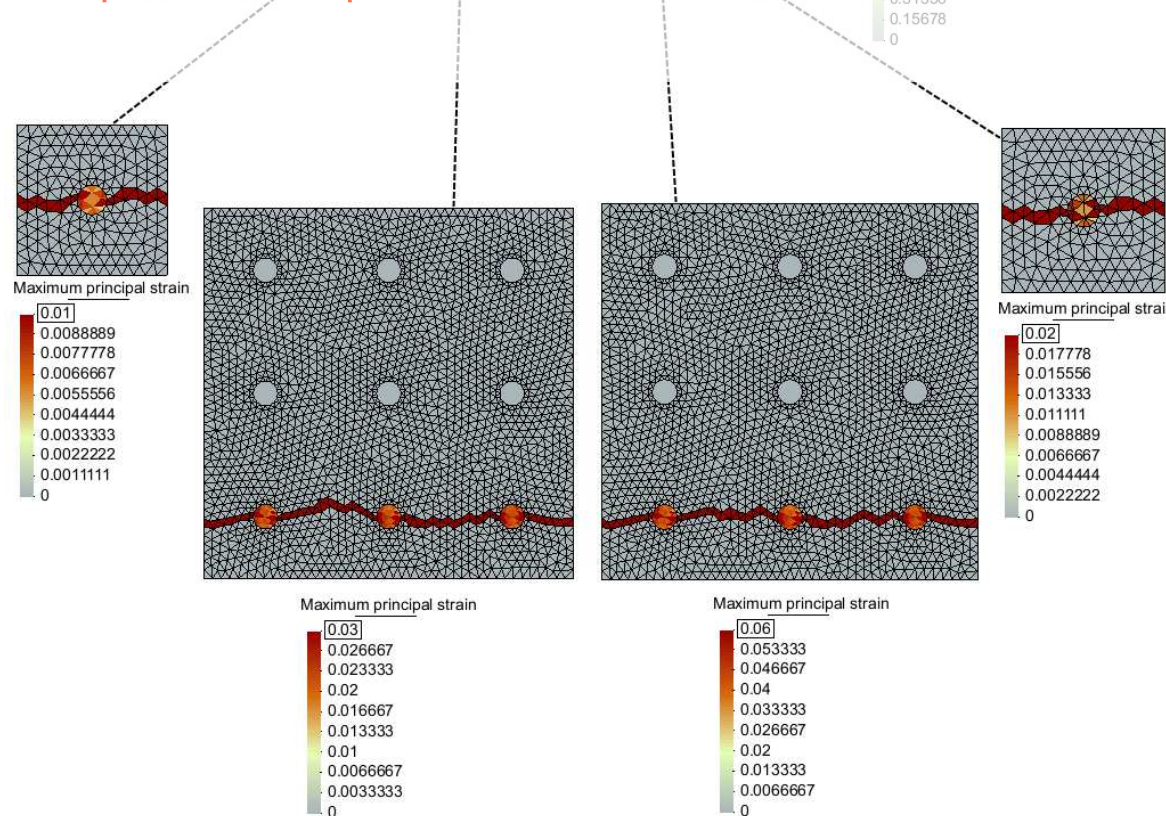

Fig. 8: macro-scale displacement field - RVEs maximum principal strain field (with regularization) 


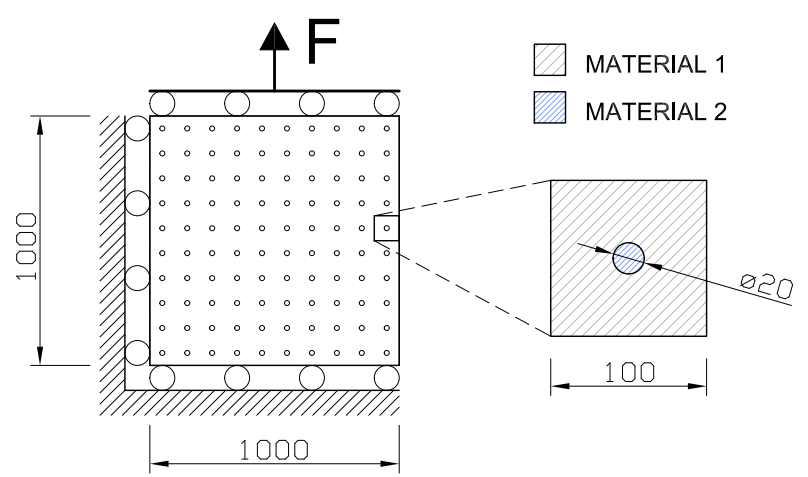

Fig. 4: geometry of the model and boundary conditions tion (DNS) has been performed to check the capability of the constitutive models to emulate the main behaviors of the masonry wall, and the results are discussed in 5.3. Finally the results obtained from the multiscale simulations are compared with both the experimental results and the DNS results in 5.4.

The analysis has been performed using Kratos Multiphysics [14, 15], a free open-source framework for the development of multidisciplinary solvers, developed at CIMNE, while pre and post-processing have been done in $\mathrm{GiD}$ [1], also developed at CIMNE.

\subsection{Experimental test: TU Eindhoven shear wall}

The geometry of the wall (here denoted as W1) as well as the boundary conditions are represented in Figure 9. The wall has no opening and it is made of one layer of solid bricks with dimensions $210 \mathrm{~mm} \times 52 \mathrm{~mm} \times$ $100 \mathrm{~mm}$, with $10 \mathrm{~mm}$ thick mortar joints [48]. The wall

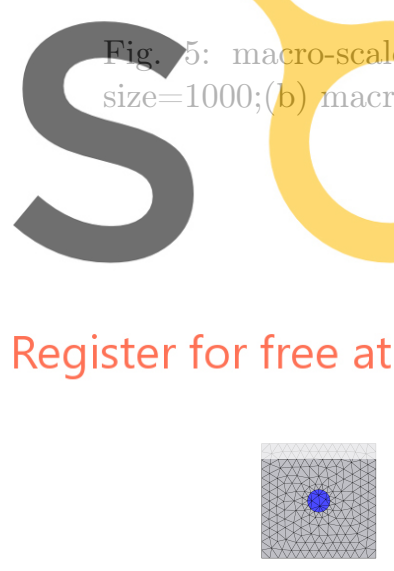

(a)

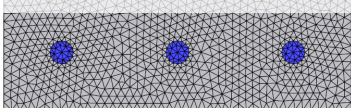

(b)
Fig. 6: (a)RVE 1x1- size=100; (b)RVE 3x3 - size=300

\section{Application to masonry structures. Numerical modeling of shear walls}

In this section, the proposed method is used to simulate the in-plane behavior of masonry structures. Shear walls has been commonly used as in-plane shear tests. Here we consider the experimental results obtained in [48] as a reference to assess the capability of the proposed approach. First, a brief description of the selected experimental test is given in 5.1, then the adopted RVE and the constitutive models used for each micro-structural constituent are described in 5.2. Before proceeding with the multiscale simulation, a Direct Numerical Simula-

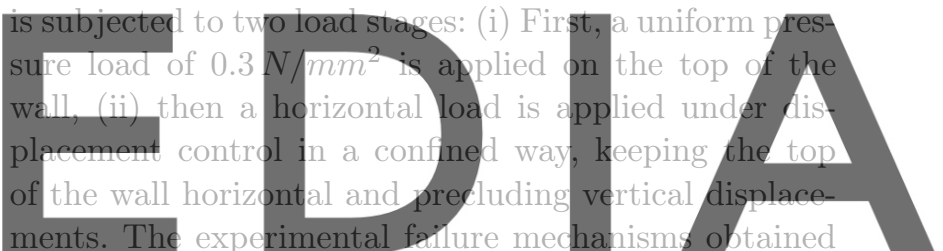

from the tested walls are shown in Figure 10.

\subsection{RVE and micro-scale constitutive laws}

The walls considered in this section are made of running bond masonry, thus showing a periodic texture. Taking advantage of the periodicity of the micro-structure, periodic boundary displacement fluctuations (see Eq. (8)) are enforced on the boundaries of the RVE. In some applications, the assumption of periodic boundary fluctuations might enforce unphysical constraints on the crack direction [13]. However, for the specific case of masonry the cracks mainly take place following the periodic geometry of the weak mortar joints. In this sense, the periodic assumption does not spoil the actual cracking direction, both in the case of failure of bed/head joints and in the case of staircase cracks. The mesh for both bricks and mortar joints consists in a structured grid of standard displacement-based 4-node quadrilateral plane stress elements, with a uniform size of $10.0 \mathrm{~mm}$, as shown in Figure 11. 


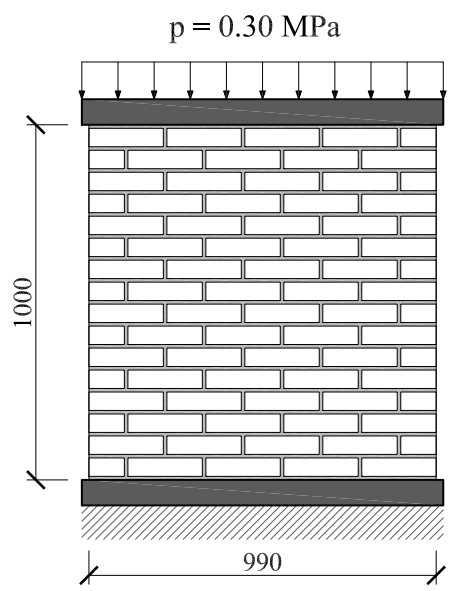

(a)

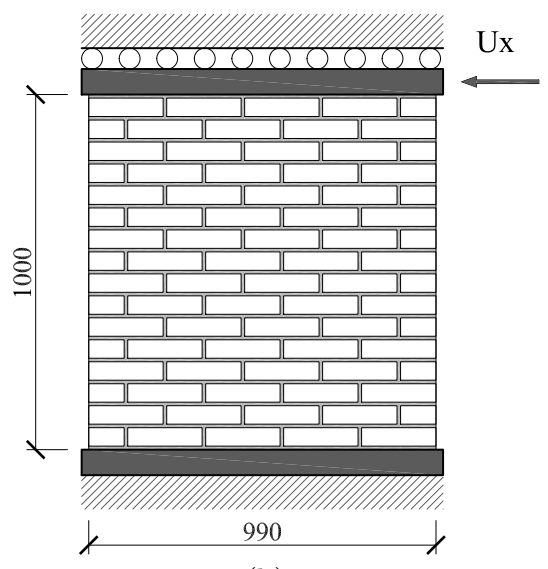

(b)

Fig. 9: Wall W1 - TU Eindhoven shear wall [48]. Geometry and loading stages: (a) stage 1: uniform vertical load; (b) stage 2: horizontal displacement under vertical confinement

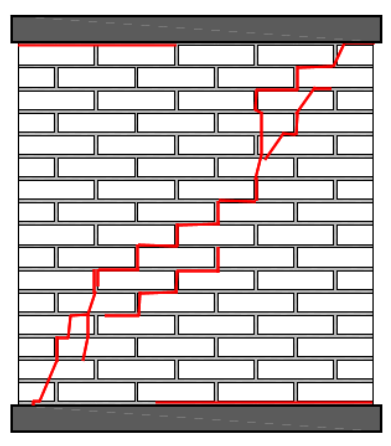

(a)

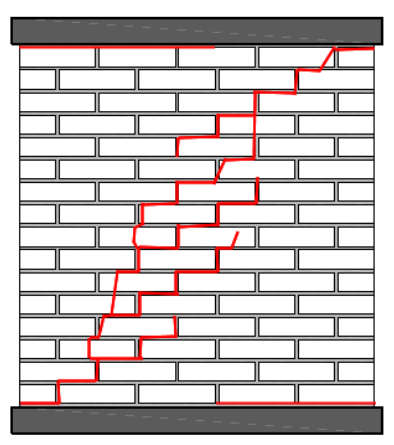

(b)
Fig. 10: experimental crack patterns

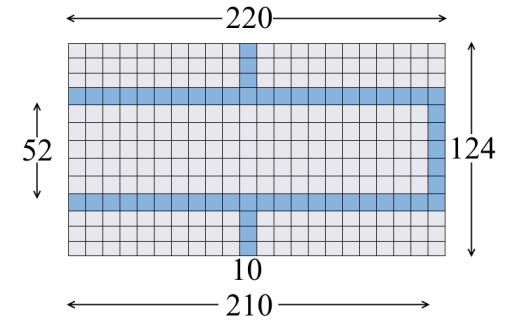

Fig. 11: RVE mesh. All units are in mm

The constitutive models used for the micro-structural constituents are here briefly presented, and they have been used for both the Direct Numerical Simulation and the Multiscale Computational Homogenization. Both constituents, bricks and mortar joints, are assumed to be isotropic. Their nonlinear behavior is simulated by means of a local continuum damage model that is an improvement of the scalar isotropic damage model described in Section 4. To take into account the different behaviors of these materials in tension and compression, a bi-dissipative $d^{+} / d^{-}$damage model has been adopted, following the work in [53], such that the stress state can be computed as

$\boldsymbol{\sigma}=\left(1-d^{+}\right) \overline{\boldsymbol{\sigma}}^{+}+\left(1-d^{-}\right) \overline{\boldsymbol{\sigma}}^{-}$

where $\overline{\boldsymbol{\sigma}}^{+}$and $\overline{\boldsymbol{\sigma}}^{-}$are the positive and negative parts of the effective stress tensor $\overline{\boldsymbol{\sigma}}$ :

$\bar{\sigma}=C: \varepsilon$

$\overline{\boldsymbol{\sigma}}^{+}=\sum_{i=1}^{3}\left\langle\bar{\sigma}_{i}\right\rangle \boldsymbol{p}_{i} \otimes \boldsymbol{p}_{i}$

$\overline{\boldsymbol{\sigma}}^{-}=\overline{\boldsymbol{\sigma}}-\overline{\boldsymbol{\sigma}}^{+}$

$d^{+}$and $d^{-}$are respectively the tensile and compressive damage indexes, and they affect respectively the positive $\overline{\boldsymbol{\sigma}}^{+}$and negative $\overline{\boldsymbol{\sigma}}^{-}$part of the effective stress tensor $\overline{\boldsymbol{\sigma}}$. Two scalar measures are introduced, termed as equivalent stresses $\tau^{+}$and $\tau^{-}$, in order to identify "loading", "unloading" or "reloading" situations for a general state of stress. In the present work we have chosen different damage surfaces than those adopted in [53]. More specifically, the same form of damage surface, based on the one proposed in [28], has been used for both tensile and compressive states, but with slight differences, in order to better capture the shear behavior of masonry components (especially mortar joints). 
The equivalent stresses $\tau^{+}$and $\tau^{-}$are computed as

$\tau^{-}=\frac{1}{1-\alpha}\left(\alpha I_{1}+\sqrt{3 J_{2}}+\kappa_{1} \beta\left\langle\sigma_{\max }\right\rangle\right)$

$\tau^{+}=\frac{1}{1-\alpha}\left(\alpha I_{1}+\sqrt{3 J_{2}}+\beta\left\langle\sigma_{\max }\right\rangle\right) \frac{f_{t}}{f_{c p}}$

$\alpha=\frac{\kappa_{b}-1}{2 \kappa_{b}-1}$

$\beta=\frac{f_{c p}}{f_{t}}(1-\alpha)-(1+\alpha)$

where $I_{1}$ is the first invariant of the stress tensor, $J_{2}$ is the second invariant of the stress deviator, $\sigma_{\max }$ is the maximum principal stress, and $\kappa_{b}$ is the ratio between bi-axial and uniaxial compressive strengths. The constant $\kappa_{1}$ in Eq. (50) has been here introduced to better control the effect of the compressive surface on the shear strength of the model. This constant can range from 0 (leading to the Drucker-Prager criterion) to 1 (leading to the criterion proposed in [28]). $f_{t}$ and $f_{c p}$ are respectively the tensile and compressive peak stresses, but while $f_{t}$ coincides with the initiation of non linearity in uniaxial tension, $f_{c p}$ doesn't coincide with the initiation of non linearity in uniaxial compression $f_{c 0}$, due to the initial hardening. This choice is motivated by the fact that in tension no hardening has been considered. Finally the term $\frac{f_{t}}{f_{c p}}$ has been introduced in Eq. (51) to relate $\tau^{+}$to the tensile strength $f_{t}$.

Being the damage an irreversible process, other two scalar quantities are introduced, termed as damage thresholds $r^{ \pm}$, that represent the largest values ever reached by the equivalent stresses $\tau^{ \pm}$during the loading history. The damage thresholds $r^{ \pm}$at time $t+\Delta t$ can be evaluated as

$r^{ \pm}=\max \left(r_{0}^{ \pm}, \max \left(r_{t}^{ \pm}, \tau^{ \pm}\right)\right)$

$r_{0}^{+}=f_{t}$

$r_{0}^{-}=f_{c 0}$

where $r_{0}^{+}$and $r_{0}^{-}$represent the initial damage thresholds respectively in tension and compression (i.e. the elastic limits in uniaxial tension $f_{t}$ and compression

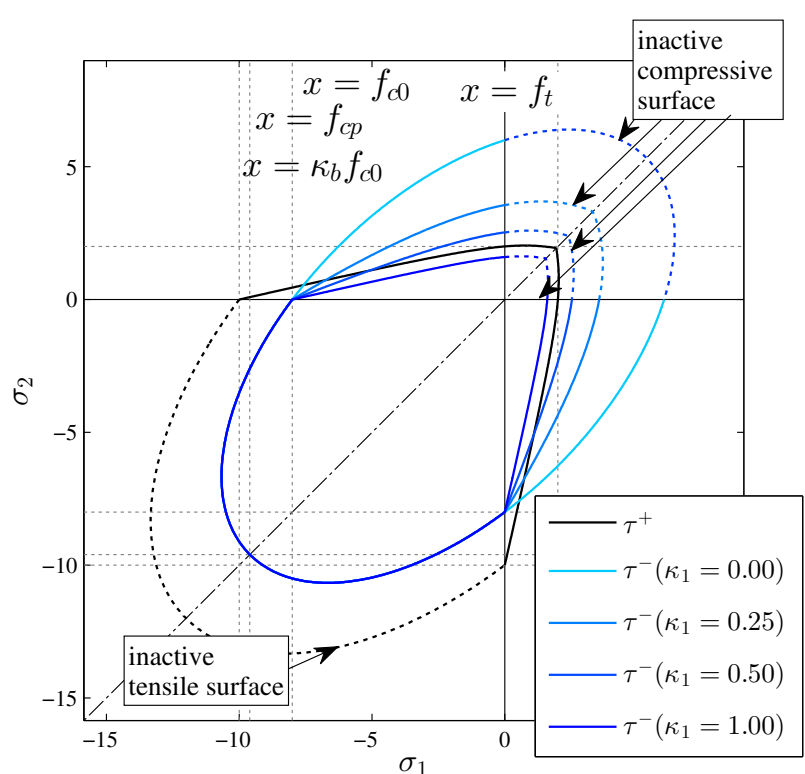

Fig. 12: initial damage surfaces (2D plane-stress)

$\left.f_{c 0}\right)$. Accordingly, the following damage criteria are introduced:

$\Phi\left(\tau^{ \pm}, r^{ \pm}\right)=\tau^{ \pm}-r_{t}^{ \pm} \leq 0$

A plot with the superposition of the two damage surfaces, in principal stress space for the $2 \mathrm{D}$ plane-stress case, is given in Figure 12. The compressive surface is plotted with different values of the constant $\kappa_{1}$. Since these surfaces are defined for any stress state, it is necessary to make them inactive (as pointed out in Figure 12 with dashed lines) under certain conditions:

1. compressive surface is allowed to evolve only if at least one principal stress is negative

2. tensile surface is allowed to evolve only if at least one principal stress is positive

The tensile damage index $d^{+}$is calculated according to the following equation:

$d^{+}\left(r^{+}\right)=1-\frac{r_{0}^{+}}{r^{+}} \exp \left\{2 H_{d i s}\left(\frac{r_{0}^{+}-r^{+}}{r_{0}^{+}}\right)\right\}$

where $H_{d i s}$ is the discrete softening parameters, and it's calculated as explained in Eq. (45). The resulting tensile uniaxial law is given in Figure 13.

For the evaluation of the compressive damage index $d^{-}$an ad hoc formulation has been adopted, in order 


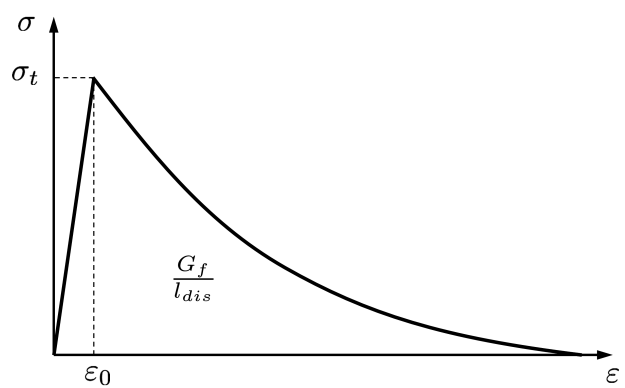

Fig. 13: tensile uniaxial law

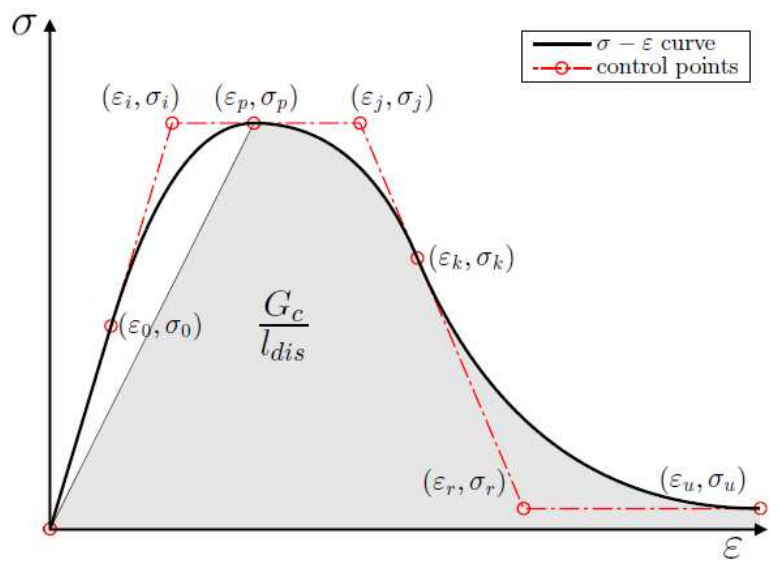

Fig. 14: compressive uniaxial law

to obtain a compressive uniaxial law like the one given in Figure 14.

This curve consists of: 1$)$ a linear part $\left[(0,0)-\left(\varepsilon_{0}, \sigma_{0}\right)\right]$, 2) a hardening $\left.\operatorname{part}\left[\left(\varepsilon_{0}, \sigma_{0}\right)-\left(\varepsilon_{p}, \sigma_{p}\right)\right], 3-4\right)$ two softening parts $\left[\left(\varepsilon_{p}, \sigma_{p}\right)-\left(\varepsilon_{k}, \sigma_{k}\right)\right]\left[\left(\varepsilon_{k}, \sigma_{k}\right)-\left(\varepsilon_{u}, \sigma_{u}\right)\right]$, and $5)$ a final constant residual stress part $\left[\left(\varepsilon_{u}, \sigma_{u}\right)-\left(+\infty, \sigma_{u}\right)\right]$ Parts 2,3 and 4 are quadratic bezier curves. Each one has three control points defining the shape of the curve, the end-positions, and the tangents to the curve at the end-positions.

As for the tensile case, in the discrete problem this uniaxial curve needs to be regularized in such a way that the shaded area under the curve be $G_{c} / l_{d i s}$, where in the present work $l_{d i s}$ is equal to the modified characteristic length $\widetilde{l}_{c h, \mu}$, as defined in Eq. (32).

Material properties for bricks and mortar joints, used next in the DNS and Multiscale simulations, are summarized in Table 2. These values are taken from the analysis conducted in [26, 27]. The material parameters governing the compressive behavior of mortar joints have been adapted to obtain the correct compressive behavior of the masonry composite material. In fact in this simulation a plane stress behavior has been adopted, which cannot capture the typical increase in compressive capacity of mortar joints, due to the confining effect of the surrounding bricks. Another possibility of cope with this issue could be the Generalized Plane State model [2].

\begin{tabular}{cccc}
\hline Property & Bricks & Mortar Joints & Units \\
\hline \hline$E$ & 16700.0 & 800.0 & $N / \mathrm{mm}^{2}$ \\
\hline$\nu$ & 0.15 & 0.15 & - \\
\hline$\sigma_{t}$ & 2.0 & 0.25 & $\mathrm{~N} / \mathrm{mm}^{2}$ \\
\hline$G_{t}$ & 0.08 & 0.016 & $\mathrm{~N} / \mathrm{mm}$ \\
\hline$\sigma_{0}$ & 3.0 & 2.0 & $\mathrm{~N} / \mathrm{mm}^{2}$ \\
\hline$\sigma_{p}$ & 11.5 & 8.5 & $\mathrm{~N} / \mathrm{mm}^{2}$ \\
\hline$\sigma_{r}$ & 2.0 & 2.0 & $\mathrm{~N} / \mathrm{mm}^{2}$ \\
\hline$G_{c}$ & 1.0 & 80.0 & $\mathrm{~N} / \mathrm{mm}^{2}$ \\
\hline$\varepsilon_{p}$ & 0.003 & 0.04 & - \\
\hline$k_{b}$ & 1.2 & 1.6 & - \\
\hline$k_{1}$ & 0.0 & 0.16 & - \\
\hline
\end{tabular}

Table 2: Material properties for bricks and mortar joints

\subsection{Direct Numerical Simulation}

In this section, the results obtained from the DNS are discussed and compared to the experimental results. This allows to assess the capability of the constitutive model described in 5.2 to represent the in-plane behavior of shear-walls. The discretization consists in a structured mesh of standard displacement-based 4-node quadrilateral plane stress elements. The size of the discretization is $10 \mathrm{~mm}$. The nonlinear static analysis is conducted in two stages:

1. In the first stage, a uniform pressure of $0.3 \mathrm{~N} / \mathrm{mm}^{2}$ is applied on top of the wall, under load control;

2. In the second stage, a horizontal nodal load is applied on the top-right corner of the wall, under displacement control. In this second stage, the vertical displacement on top of the wall, is kept fixed at the value reached at the previous step.

Figure 15 shows the structural response in terms of horizontal top displacement against total horizontal reaction. The results obtained with the DNS are in good agreement with the experimental results, also in terms of the obtained failure mechanisms, as it can be seen in Table 3 and Table 4. 


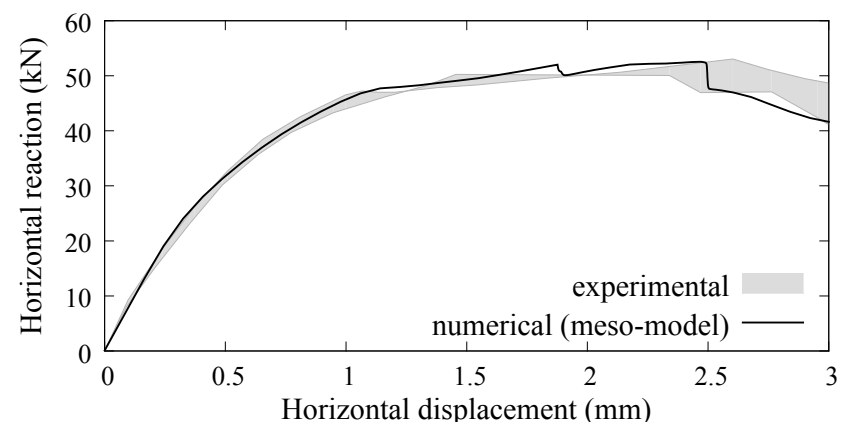

Fig. 15: Load-Displacement curve

In this context, it is interesting to highlight the main features characterizing the behavior of this kind of experiment (for a detailed description see [27]). To this end, five significant snapshots of the analysis have been extracted. These snapshots are identified by horizontal top displacements $U_{x}=[0.5,1.1,1.8,2.5,3.0] \mathrm{mm}$. The obtained results can be seen in Table 3 and Table 4 , in terms of displacement, maximum principal strain, minimum principal stress, tensile damage and compressive damage.

The first non-linearity appears at a horizontal top displacement $U_{x}=0.5 \mathrm{~mm}$, with horizontal tensile cracks developing at the bottom-right and top-left parts of the wall. In this first stage, compressive stresses form several parallel struts oriented along the direction given by the central points of the bricks. At a horizontal-topdisplacement $U_{x}=1.1 \mathrm{~mm}$, staircase diagonal cracks appear from the center of the wall, proceeding towards the supports through the mortar joints. In this stage there are several cracks, but not yet a fully open unique crack, due to the presence of bricks that are still below their tensile peak stress. The formation of these diagonal cracks also produces the first visible change in the slope of the load-displacement curve. The stage between horizontal-top-displacements $U_{x}=1.8 \mathrm{~mm}$ and $U_{x}=2.5 \mathrm{~mm}$ is characterized by the previously mentioned cracks joining to form a unique diagonal crack that fully reaches the supports. The joining of those cracks is due to tensile failure of bricks. In this stage, is also visible the change in compressive stresses, now rotating and forming two separated struts. The further opening of the diagonal crack leads to the full development of the shear mechanism for the ultimate displacement $U_{x}=3.0 \mathrm{~mm}$.

\subsection{Multiscale simulation}

In order to assess the proposed method, the multiscale homogenization analysis has been performed on four different meshes, here denoted as mesh $7 \times 7$, mesh $8 \times 8$, mesh 9x9, and mesh $15 \times 15$, as shown in Figure 16. All macro-scale models consists of a structured mesh of 4node quadrilateral plane-stress finite elements. In order to improve the performance of these rather coarse discretizations, the Enhanced Assumed Strain (EAS) quadrilateral element proposed in [51] was used.

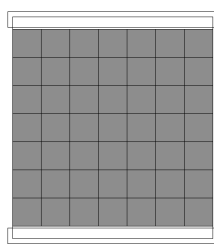

(a) $\operatorname{mesh} 7 \times 7$

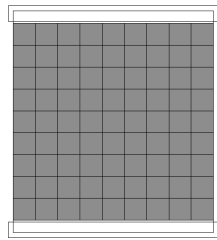

(c) mesh $9 \times 9$

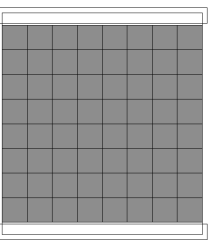

(b) mesh $8 \times 8$

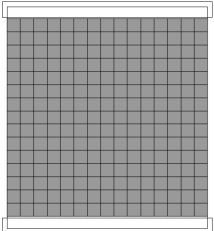

(d) mesh $15 \times 15$
Fig. 16: adopted meshes at the macro-scale

The results obtained from all four analyses are in very good agreement with the DNS predictions. Figure 17 shows how the obtained load-displacement curves closely follow the curve of the DNS. Furthermore it can be seen how upon mesh refinement the relative error among the curves diminishes, thus showing objectivity of the response thanks to the regularization procedure proposed by this research. The coarsest mesh $(7 \times 7)$ shows a premature failure in the compressed corners probably due to the sudden loss of bearing capacity of the few elements involved in the nonlinear process. However, already the $8 \times 8$ mesh shows a better agreement in the

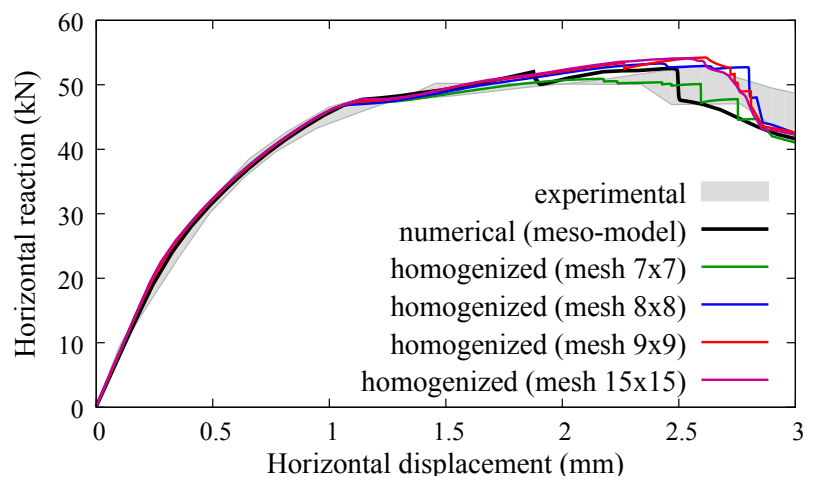

Fig. 17: Load-Displacement curve 


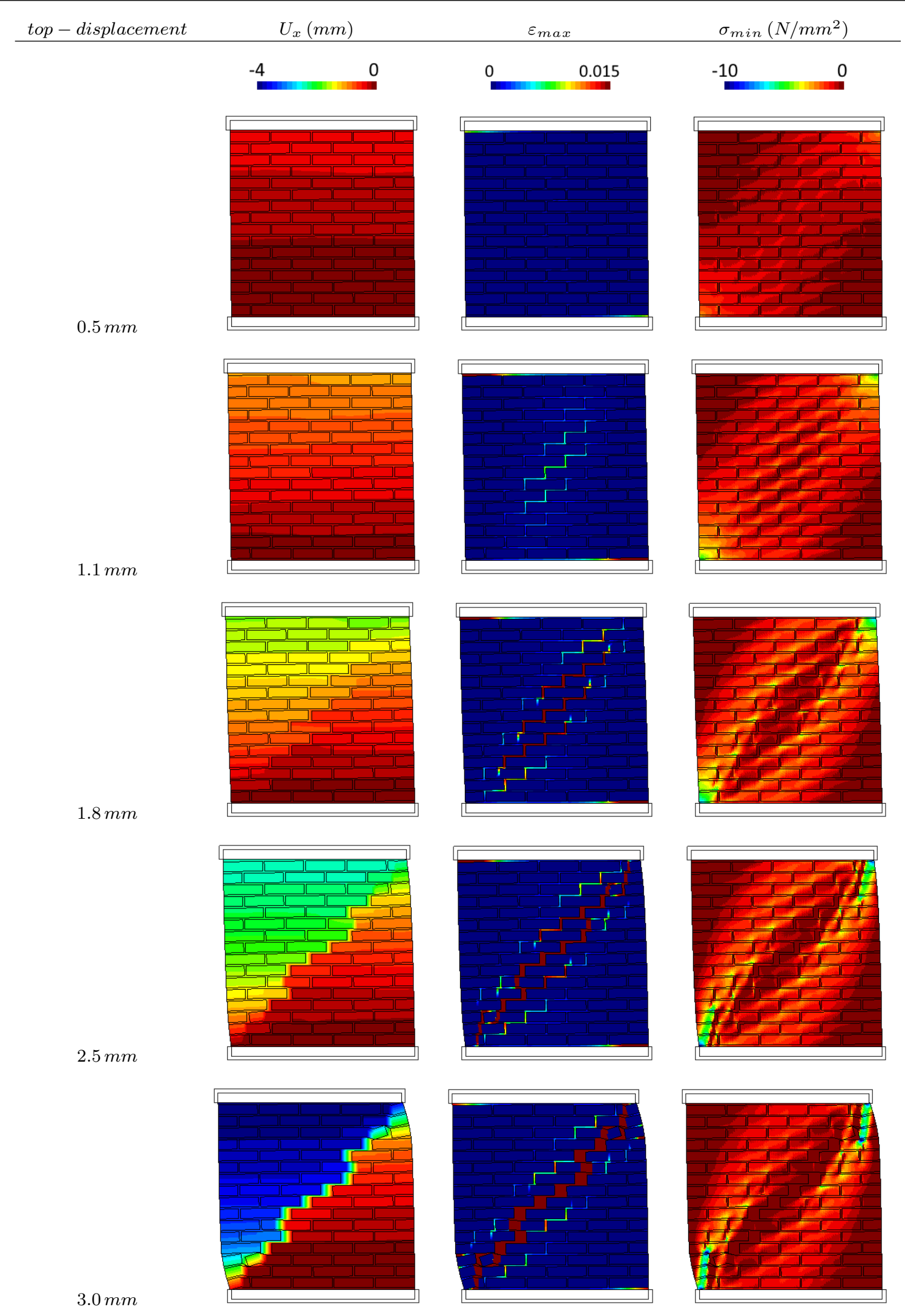

Table 3: DNS. Incremental contour plots of $\left(U_{x}\right)$ horizontal top displacement, $\left(\varepsilon_{\max }\right)$ maximum principal strain, and $\left(\sigma_{\min }\right)$ minimum principal stress 


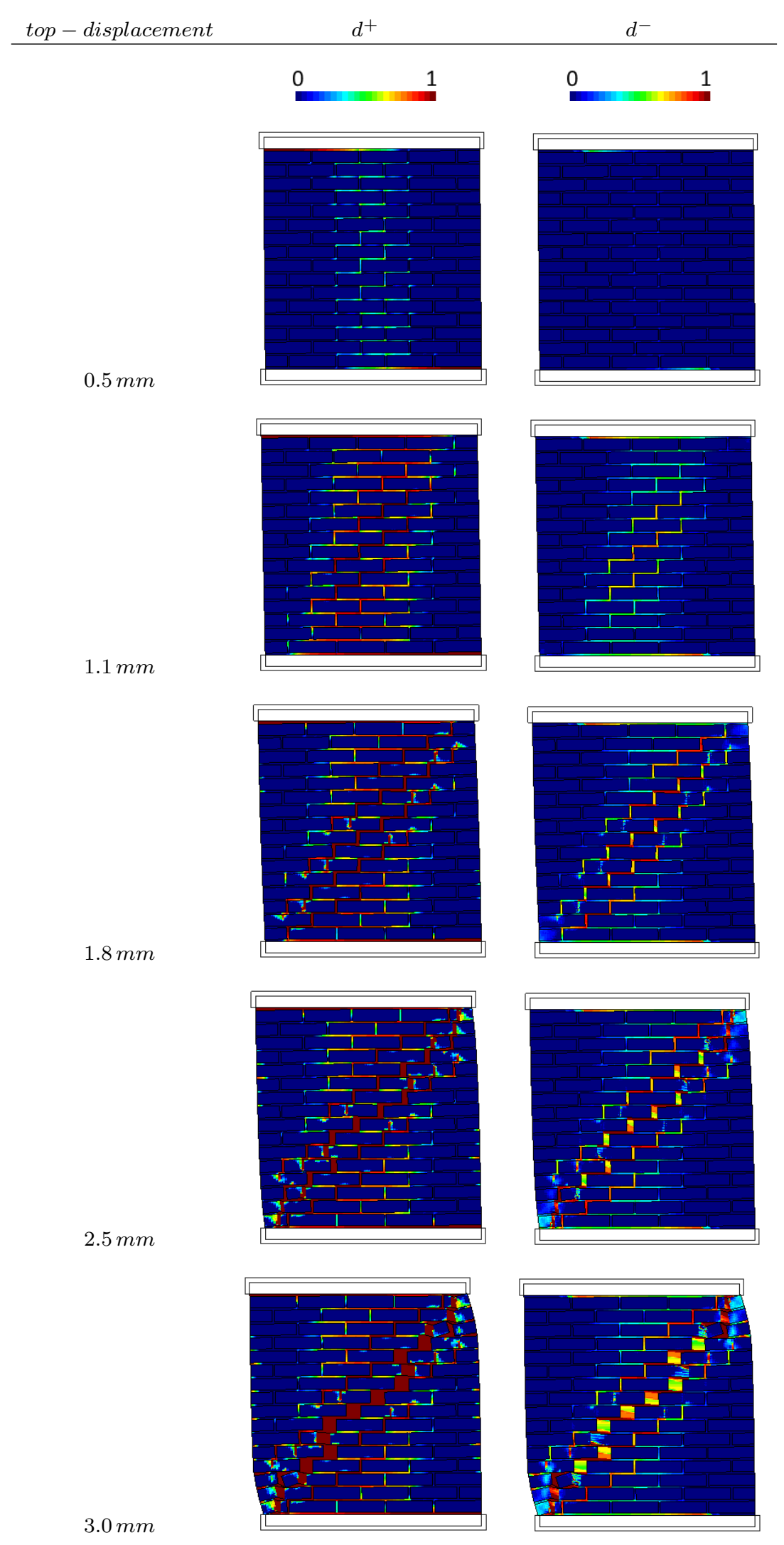

Table 4: DNS. Incremental contour plots of $\left(d^{+}\right)$tensile damage, and $\left(d^{-}\right)$compressive damage 
final stage, and finally the $9 \times 9$ and $15 \times 15$ meshes start showing smoother transitions towards the compressive failure.

The $15 \times 15$ mesh from a practical point of view may not seem the best choice for the analyzed problem, since the FE size at the macro-level is smaller than the RVE size. However, the $15 \times 15$ case has been intentionally addressed to show the mesh insensitivity of the proposed regularization method. All the other cases with coarser meshes actually look more appropriate, since the FE size at the macro-level is larger than the RVE size. In those cases, the main failure mechanisms, displacement and force capacities are correctly represented despite the mesh coarseness.

Table 6 shows the obtained results in terms of displacement, maximum principal strain and minimum principal stress. In particular, it can be seen how the shape of the crack (in terms of maximum principal strains) and the redistribution of compressive stresses closely resembles those obtained with the DNS.

Table 5 shows the final state (in terms of maximum principal strain, tensile and compressive damage) of three significant RVEs. The first RVE (RVE 1) shows a prevailing mode I failure, where the tensile damage concentrates in one of the bed joints. This is the first source of non-linearity that appears during the test, while the vertical confinement is still low. The second RVE (RVE 2) shows the typical staircase failure due to shear with low confining pressure. This RVE is taken from the center of the wall, where the main diagonal crack starts. Finally the third RVE (RVE 3 ) is taken from the bottom-left corner of the wall, where the main diagonal crack ultimately triggers the collapse of the wall. This failure happens at a later stage of the test, and due to the fact that vertical displacements on top of the wall are constrained, this area is subjected to significant concentration of compressive stresses. The RVE successfully shows a first staircase damage pattern, followed by the failure of the brick by vertical splitting.

The smeared crack approach adopted in this research, together with proper fracture energy regularization, has provided numerical results in remarkable agreement with the experimental cracking patterns on masonry shear walls.

However, it must be noted that in general the proposed regularization cannot solve (and neither attempts to) the well-known problem of mesh-bias dependency of the FE solution, that is, some preferred directions might still exist in the model depending on the orientation of the mesh. Such issue can be however addressed by carefully choosing the finite element model employed in the coarse mesh, as demonstrated by recent works $[7,10,11,12,38]$. In particular the element formulation employed in current work (EAS [51]) makes it reasonably insensitive to mesh bias.

\subsection{Computational cost}

An important aspect in this kind of simulations is the computational cost. Even if such a study is out of the scope of this paper, this section gives just a brief comparison between the DNS and the Multiscale simulations. It should be noted that the specimen described in 5.1 is quite small $(1 \mathrm{~m} \times 1 \mathrm{~m})$ and it is not the perfect candidate for a multiscale simulation. Nevertheless it has been analyzed for a comparison with experimental results. To exploit the advantages of the proposed multiscale model, a second DNS has been conducted on a larger model $(3 m \times 3 m)$ which is a scaled version of the original one. For both cases a multiscale simulation has been carried out, using the same macro-scale discretization $(7 \times 7$ mesh) which proved to be sufficient to obtain good results in both analysis (see Figure 18a and Figure 18b). Table 7a and Table $7 \mathrm{~b}$ show the computational cost of the DNS and the Multiscale simulations in terms of memory usage and (real) time. To give an

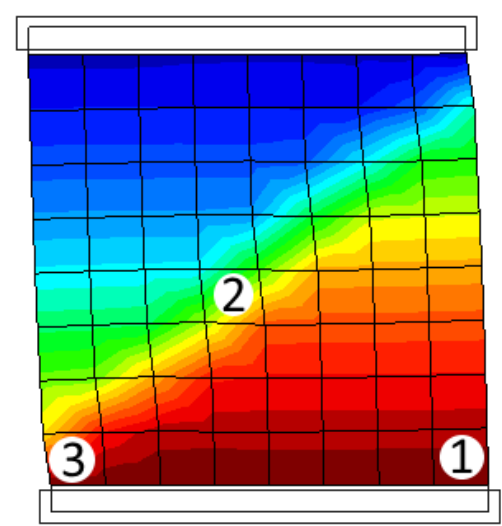

RVE

$\varepsilon_{\max }$ $d^{+}$ $d^{-}$

RVE 1
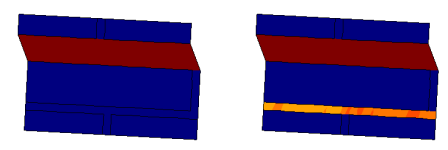

RVE 2
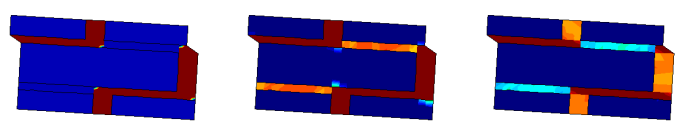

RVE 3
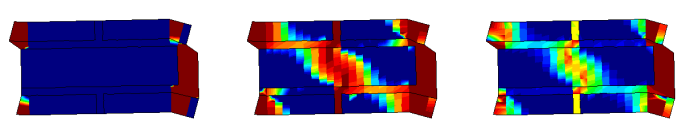

Table 5: final state in three significant RVEs 


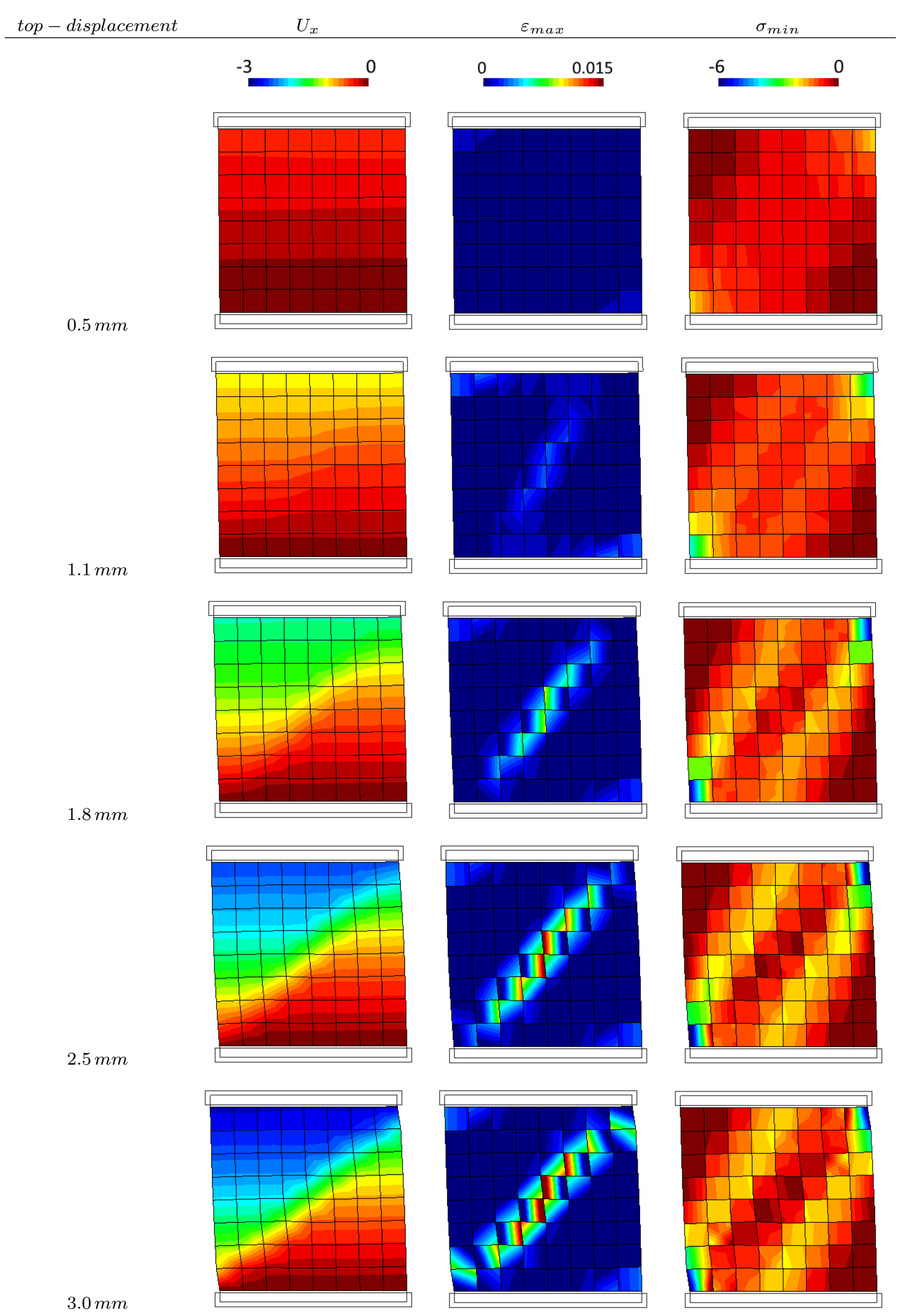

Table 6: CHM. Incremental contour plots of $\left(U_{x}\right)$ horizontal top displacement, $\left(\varepsilon_{\max }\right)$ maximum principal strain, and $\left(\sigma_{\min }\right)$ minimum principal stress 


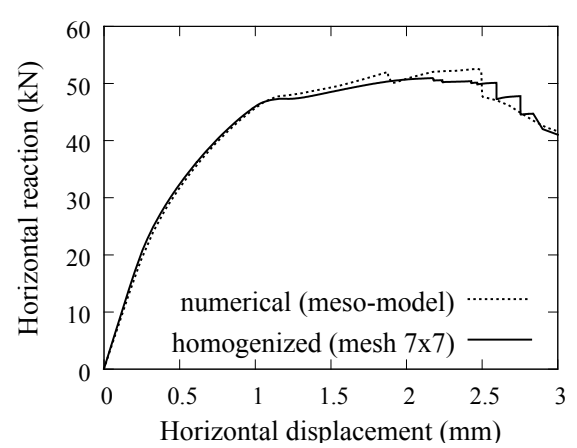

(a)

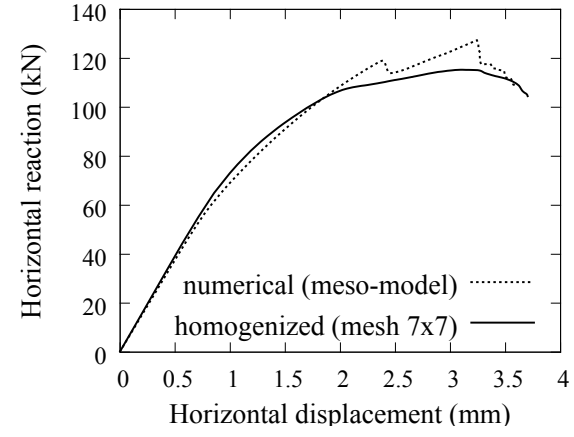

(b)

Fig. 18: load-displacement curves for the original (a) and scaled model (b)

\begin{tabular}{ccccc}
\hline DNS model & $\begin{array}{c}\text { number } \\
\text { of nodes }\end{array}$ & $\begin{array}{c}\text { number } \\
\text { of } \\
\text { elements }\end{array}$ & $\begin{array}{c}\text { average } \\
\text { time per } \\
\text { iteration }\end{array}$ & memory \\
\hline \hline $1 m \times 1 m$ & 11152 & 10934 & 1.51 sec. & $120 \mathrm{MB}$ \\
\hline $3 m \times 3 m$ & 77064 & 76502 & 9.72 sec. & $730 \mathrm{MB}$ \\
\hline
\end{tabular}

(a)

\begin{tabular}{ccccccc}
\hline $\begin{array}{c}\text { number } \\
\text { of } \\
\text { nodes }\end{array}$ & $\begin{array}{c}\text { number } \\
\text { of } \\
\text { elements }\end{array}$ & $\begin{array}{c}\text { number } \\
\text { of RVEs }\end{array}$ & $\begin{array}{c}\text { number } \\
\text { of nodes } \\
\text { (RVE) }\end{array}$ & $\begin{array}{c}\text { number } \\
\text { of } \\
\text { elements } \\
\text { (RVE) }\end{array}$ & $\begin{array}{c}\text { average } \\
\text { time } \\
\text { per it- } \\
\text { eration }\end{array}$ & memory \\
\hline \hline 64 & 49 & 196 & 322 & 356 & 4.97 sec. & $275 \mathrm{MB}$ \\
\hline
\end{tabular}

(b)

Table 7: computational cost for the Direct Numerical Simulations (a) and for the Multiscale simulation with a 7x7 mesh (b)

objective comparison, the measured time is not referred to the entire simulation, but to the average duration of a single global iteration (i.e. the total duration of a load step, divided by the number of equilibrium iterations). Furthermore the time has been measured during specific load steps where almost the entire model is in a nonlinear range.

As it can be observed, the multiscale simulation proves to be efficient with respect to the DNS when dealing with large structures (or alternatively when the microstructure is very small compared to the specimen). The DNS is penalized when used with large models due to increasing time and memory requirements, mainly required by the storage and solution of large linear systems of equations. On the contrary, the Multiscale simulation is penalized when used with small models, where the overhead of the homogenization process dominates.

All the computations have been done on a single machine with an Intel Core i7-2670QM-2.20GHz CPU and
8.00 GB RAM. The code has been parallelized using OpenMP.

\section{Conclusions}

Classical first order computational homogenization is a well established framework used to capture the effect that a complex micro-structure has on the structural level. It has been however recognized to suffer from spurious dependence on the macro-mesh size as well as on the RVE size, when strain localization takes place.

In this paper, the authors propose a simple regularization technique that allows to use classical first order computational homogenization for problems involving strain localizations, while keeping its settings, and thus its simplicity, unchanged. In the context of the Smeared Crack Approach (SCA), the Fracture-Energybased regularization, commonly used in 1-scale FE analysis when dealing with strain-softening materials, is ex- 
tended here to 2-scale FE analysis. The proposed regularization procedure ensures objectivity of the dissipation at the macro-scale, by embedding in the definition of the micro-scale characteristic lengths (used to regularize micro-structural constitutive laws), the size of the finite elements from both scales as well as the size of the RVE itself. This is achieved by means of a scaling factor which is constant over the RVE. Of particular interest is the fact that an existing FE code implementing classical computational homogenization needs only a marginal change in the evaluation of this scaling factor.

The proposed method is validated through basic benchmark tests as well as with more involved analyses of inplane loaded masonry shear walls, showing satisfactory results. More specifically, the application of the proposed multiscale procedure shows a remarkable agreement with the results given by the DNS. As for the analysis of the masonry shear wall, the proposed model has shown its capability at the micro-scale to represent the local tensile, compressive and shear failures due to the complex interaction between units and joints. At the macro-scale, the model can correctly describe the phenomenological structural response. The proposed multiscale approach is robust and objective for different mesh discretizations.

Acknowledgements This research has received the financial support from the Graduate School of the University "G. D'Annunzio" of Chieti-Pescara, from the Italian Department of Civil Protection through the Reluis Project, from the MINECO (Ministerio de Economia y Competitividad of the Spanish Government) and the ERDF (European Regional Development Fund) through the MICROPAR project (Identification of mechanical and strength parameters of structural masonry by experimental methods and numerical micro-modelling, ref num. BIA2012-32234) and from the Excellence Programme for Knowledge Generation by MINECO, through the EACY project (Enhanced accuracy computational and experimental framework for strain localization and failure mechanisms, ref. MAT2013-48624-C2-1-P).

S. Oller acknowledges the support of the European Research Council under the Advanced Grant: ERC-2012-AdG 320815 COMP-DES-MAT "Advanced tools for computational design of engineering materials"

\section{References}

1. (2002) Gid: The personal pre and post preprocessor

2. Addessi D, Sacco E (2014) A kinematic enriched plane state formulation for the analysis of masonry panels. European Journal of Mechanics-A/Solids 44:188-200

3. Addessi D, Sacco E, Paolone A (2010) Cosserat model for periodic masonry deduced by nonlinear homogenization. European Journal of MechanicsA/Solids 29(4):724-737

4. Badillo HA (2012) Numerical modelling based on the multiscale homogenization theory. application in composite materials and structures. $\mathrm{PhD}$ thesis, Universitat Politécnica de Catalunya BarcelonaTech

5. Bažant Z, Oh B (1983) Crack Band Theory for Fracture of Concrete. Bordas-Dunod, URL http: //books.google.it/books? id=x9citwAACAAJ

6. Belytschko T, Loehnert S, Song JH (2008) Multiscale aggregating discontinuities: a method for circumventing loss of material stability. International Journal for Numerical Methods in Engineering 73(6):869-894

7. Benedetti L, Cervera M, Chiumenti M (2015) Stress-accurate mixed fem for soil failure under shallow foundations involving strain localization in plasticity. Computers and Geotechnics 64:32-47

8. Bosco E, Kouznetsova V, Geers M (2015) Multiscale computational homogenization-localization for propagating discontinuities using $\mathrm{x}$-fem. International Journal for Numerical Methods in Engineering 102(3-4):496-527

9. Car E, Zalamea F, Oller S, Miquel J, Oñate E (2002) Numerical simulation of fiber reinforced composite materials - two procedures. International journal of solids and structures 39(7):19671986

10. Cervera M, Chiumenti M (2006) Mesh objective tensile cracking via a local continuum damage model and a crack tracking technique. Computer Methods in Applied Mechanics and Engineering 196(1):304-320

11. Cervera M, Chiumenti M, Codina R (2010) Mixed stabilized finite element methods in nonlinear solid mechanics. part ii: Strain localization. Computer Methods in Applied Mechanics and Engineering 199(37):2571-2589

12. Cervera M, Pelà L, Clemente R, Roca P (2010) A crack-tracking technique for localized damage in quasi-brittle materials. Engineering Fracture Mechanics 77(13):2431-2450

13. Coenen E, Kouznetsova V, Geers M (2012) Novel boundary conditions for strain localization analyses in microstructural volume elements. International Journal for Numerical Methods in Engineering 90(1):1-21, DOI 10.1002/nme.3298, URL http://dx.doi.org/10.1002/nme.3298

14. Dadvand P, Rossi R, Oñate E (2010) An objectoriented environment for developing finite element codes for multi-disciplinary applications. Archives of computational methods in engineering 


\section{$17(3): 253-297$}

15. Dadvand P, Rossi R, Gil M, Martorell X, Cotela J, Juanpere E, Idelsohn SR, Oñate E (2013) Migration of a generic multi-physics framework to hpc environments. Computers \& Fluids 80:301-309

16. De Bellis ML (2009) A cosserat based multi-scale technique for masonry structures. In: PHD Thesis

17. De Bellis ML, Addessi D (2011) A cosserat based multi-scale model for masonry structures. International Journal for Multiscale Computational Engineering $9(5)$

18. Geers M, Kouznetsova VG, Brekelmans WAM (2003) Multiscale first-order and second-order computational homogenization of microstructures towards continua. International Journal for Multiscale Computational Engineering 1(4)

19. Hernández JA, Oliver J, Huespe AE, Caicedo M (2012) High-Performance Model Reduction Procedures in Multiscale Simulations. 127, Monograph CIMNE

20. Hill R (1965) A self-consistent mechanics of composite materials. J Mech Phys Solids 13:213-222

21. Hughes TJ, Feijóo GR, Mazzei L, Quincy JB (1998) The variational multiscale method-a paradigm for computational mechanics. Computer methods in applied mechanics and engineering 166(1):3-24

22. Kouznetsova V, Geers MGD, Brekelmans WAM (2002) Multi-scale constitutive modelling of heterogeneous materials with a gradient-enhanced computational homogenization scheme. International Journal for Numerical Methods in Engineering 54(8):1235-1260

23. Kouznetsova V, Geers M, Brekelmans W (2004) Multi-scale second-order computational homogenization of multi-phase materials: a nested finite element solution strategy. Computer Methods in Applied Mechanics and Engineering 193:5525-5550, advances in Computational Plasticity

24. Lloberas-Valls O, Rixen D, Simone A, Sluys L (2011) Domain decomposition techniques for the efficient modeling of brittle heterogeneous materials. Computer Methods in Applied Mechanics and Engineering 200(13):1577-1590

25. Lloberas-Valls O, Rixen D, Simone A, Sluys L (2012) Multiscale domain decomposition analysis of quasi-brittle heterogeneous materials. International Journal for Numerical Methods in Engineering 89(11):1337-1366

26. Lourenço PB, Rots JG (1997) Multisurface interface model for analysis of masonry structures. Journal of engineering mechanics 123(7):660-668

27. Lourenco PB (1996) Computational strategies for masonry structures. PhD thesis, TU Delft, Delft
University of Technology

28. Lubliner J, Oliver J, Oller S, Oñate E (1989) A plastic-damage model for concrete. International Journal of solids and structures 25(3):299-326

29. Mandel J (1971) Plasticité classique et viscoplasticité. Springer-Verlag

30. Massart T, Peerlings R, Geers M (2007) An enhanced multi-scale approach for masonry wall computations with localization of damage. International journal for numerical methods in engineering 69(5):1022-1059

31. Massart TJ (2003) Multi-scale modeling of damage in masonry structures

32. Mercatoris B, Massart T (2011) A coupled twoscale computational scheme for the failure of periodic quasi-brittle thin planar shells and its application to masonry. International journal for numerical methods in engineering 85(9):1177-1206

33. Mercatoris B, Bouillard P, Massart T (2009) Multiscale detection of failure in planar masonry thin shells using computational homogenisation. Engineering fracture mechanics 76(4):479-499

34. Neto EADS, Feijóo RA (2006) Variational foundations of multi-scale constitutive models of solid: Small and large strain kinematical formulation. LNCC Research and Development (16)

35. Nguyen VP, Stroeven M, Sluys LJ (2011) Multiscale continuous and discontinuous modeling of heterogeneous materials: A review on recent developments. Journal of Multiscale Modelling 3(04):229 270

36. Oliver J (1989) A consistent characteristic length for smeared cracking models. International Journal for Numerical Methods in Engineering 28(2):461474

37. Oliver J, Caicedo M, Roubin E, Hernández J, Huespe A (2014) Multi-scale (fe2) analysis of material failure in cement/aggregate-type composite structures. Computational Modelling of Concrete Structures p 39

38. Oliver J, Dias I, Huespe AE (2014) Crack-path field and strain-injection techniques in computational modeling of propagating material failure. Computer Methods in Applied Mechanics and Engineering 274:289-348

39. Oliver Olivella X, Caicedo Silva MA, Roubin E, Huespe AE, et al (2014) Continuum approach to computational multi-scale modeling of fracture. Key engineering materials 627:349-352

40. Oller S (2001) Fractura Mecánica: Un Enfoque Global. Ediciones CIMNE y UPC

41. Oller S, Miquel Canet J, Zalamea F (2005) Composite material behavior using a homogenization 
double scale method. Journal of Engineering mechanics 131(1):65-79

42. Ortolano JM, Hernández JA, Oliver J (2013) A Comparative Study on Homogenization Strategies for Multi-Scale Analysis of Materials. 135, Monograph CIMNE

43. Otero F, Martínez X, Oller S, Salomón O (2012) Study and prediction of the mechanical performance of a nanotube-reinforced composite. Composite Structures 94:2920-2930

44. Otero F, Oller S, Martinez X, Salomón O (2015) Numerical homogenization for composite materials analysis. comparison with other micro mechanical formulations. Composite Structures 122:405-416

45. Pelà L, Cervera M, Roca P (2011) Continuum damage model for orthotropic materials: Application to masonry. Computer Methods in Applied Mechanics and Engineering 200(9):917-930

46. Pelà L, Cervera M, Roca P (2013) An orthotropic damage model for the analysis of masonry structures. Construction and Building Materials 41:957967

47. Quinteros RD, Oller S, Nallim LG (2012) Nonlinear homogenization techniques to solve masonry structures problems. Composite Structures 94(2):724730

48. Raijmakers T, Vermeltfoort A (1992) Deformation controlled tests in masonry shear walls: report B92-1156. URL http://books.google.it/books? id=HUmTPgAACAAJ

49. Rots JG (1988) Computational modeling of concrete fracture. PhD thesis, Technische Hogeschool Delft

50. Scott MH, Fenves GL (2003) A krylov subspace accelerated newton algorithm. In: Proc., 2003 ASCE Structures Congress

51. Simo JC, Rifai M (1990) A class of mixed assumed strain methods and the method of incompatible modes. International Journal for Numerical Methods in Engineering 29(8):1595-1638

52. Trovalusci P, Masiani R (2003) Non-linear micropolar and classical continua for anisotropic discontinuous materials. International Journal of Solids and Structures 40(5):1281-1297

53. Wu JY, Li J, Faria R (2006) An energy release ratebased plastic-damage model for concrete. International Journal of Solids and Structures 43(3):583612

54. Zucchini A, Lourenço P (2002) A micro-mechanical model for the homogenisation of masonry. International Journal of Solids and Structures $39(12): 3233-3255$
55. Zucchini A, Lourenço PB (2009) A micromechanical homogenisation model for masonry: Application to shear walls. International Journal of Solids and Structures 46(3):871-886 